\title{
The value of a liability cash flow in discrete time subject to capital requirements
}

\author{
Hampus Engsner ${ }^{1} \cdot$ Kristoffer Lindensjö $^{1}$ • \\ Filip Lindskog ${ }^{1}$
}

Received: 10 August 2018 / Accepted: 3 July 2019 / Published online: 27 September 2019

(C) The Author(s) 2019

\begin{abstract}
The aim of this paper is to define the market-consistent multi-period value of an insurance liability cash flow in discrete time subject to repeated capital requirements, and explore its properties. In line with current regulatory frameworks, the presented approach is based on a hypothetical transfer of the original liability and a replicating portfolio to an empty corporate entity, whose owner must comply with repeated one-period capital requirements but has the option to terminate the ownership at any time. The value of the liability is defined as the no-arbitrage price of the cash flow to the policyholders, optimally stopped from the owner's perspective, taking capital requirements into account. The value is computed as the solution to a sequence of coupled optimal stopping problems or, equivalently, as the solution to a backward recursion.
\end{abstract}

Keywords Market-consistent valuation $\cdot$ Replicating portfolios $\cdot$ Capital requirements

Mathematics Subject Classification (2010) 91G50 • 91G20 • 91B74

JEL Classification G32 · G13 · G22

F. Lindskog

lindskog@math.su.se

H. Engsner

hampus.engsner@math.su.se

K. Lindensjö

kristoffer.lindensjo@math.su.se

1 Department of Mathematics, Stockholm University, 10691 Stockholm, Sweden 


\section{Introduction}

The aim of this paper is to define the market-consistent multi-period value of a liability cash flow in discrete time subject to repeated capital requirements in accordance with current regulatory frameworks, and explore its properties. The valuation procedure will be studied within an insurance liability context. However, the valuation procedure could be used for any liability where the debtor has limited liability and faces capital requirements.

Essentially, given an optimally selected replicating portfolio, the externally imposed capital requirements define the market-consistent value of a liability as the value it would have if it were transferred to an empty corporate entity, called a reference undertaking, whose owner has the option to terminate the ownership (limited liability, option to default).

The transfer of liabilities is a hypothetical transfer considered for the sake of valuation. In particular, possible existing liabilities of the eventual owner of the reference undertaking are not considered since it is not known at the time of valuation who the owner might be. By not considering existing liabilities of the receiver, there may be a loss of diversification benefits leading to a conservative liability value. On the other hand, the liability to be valued should be interpreted as the aggregate liability of a company, i.e., at the level on which capital requirements are imposed. Therefore, compared to the valuation of cash flows of individual contracts, which is not considered here, there may be substantial diversification benefits.

The transfer and the valuation procedure can be summarised as follows:

(1) We assume that the following items are transferred to a reference undertaking that has no other assets or liabilities: (i) the liability to be valued, (ii) an asset portfolio, called the replicating portfolio, with a cash flow meant to, at least partially, offset the liability cash flow, and (iii) an amount in a numéraire asset, which is such that the reference undertaking precisely meets the imposed capital requirement at the time of the transfer.

(2) We assume that the owner of the reference undertaking has limited liability and therefore can choose to not further finance the reference undertaking at any future time-an option to default. We assume that the reference undertaking cannot change the transferred replicating portfolio.

(3) We assume that the market is arbitrage-free in the sense that an equivalent pricing measure exists. We derive the value of the reference undertaking by identifying it with the price of a particular American-type financial derivative: the price of the optimally stopped cash flow to the owner of the reference undertaking, taking limited liability and capital requirements into account. This value is the price paid to the owner of the original insurance company for ownership of the reference undertaking. Similarly, the value of the liability is defined as the price of the cash flow to the policyholders, optimally stopped from the perspective of the owner of the reference undertaking. Due to the option to default, the cash flow the policyholders are entitled to is not identical to the cash flow they receive.

(4) The procedure outlined above results in a liability value that depends on the composition of the replicating portfolio and the capital requirements. We focus primarily on capital requirements in terms of conditional monetary risk measures such 
as value-at-risk and expected shortfall. We consider several criteria for choosing the replicating portfolio. Although the liability values obtained from the valuation procedure are market-consistent only if the replicating portfolio criterion is chosen appropriately, see Remark 2.7 , the valuation procedure does not assume a particular replicating portfolio criterion nor a particular sequence of conditional monetary risk measures.

The approach to market-consistent liability valuation presented in Möhr [19] has been the main source of inspiration for the current paper. In [19], a valuation framework based on dynamic replication and cost-of-capital arguments was presented. In Engsner et al. [10], a valuation framework inspired by [19], based on dynamic monetary risk measures and dynamic monetary utility functions was presented and explicit valuation formulas were derived under Gaussian model assumptions. An essential difference between [19] and [10] is that in the latter setup the replicating portfolio transferred to the reference undertaking together with the liability is not allowed to be modified after the transfer of the liability, throughout the run-off of the liability. The same applies to the valuation approach presented in this paper. The owner of the reference undertaking only has the option to make a decision to terminate ownership of the reference undertaking (option to default). In [10], the replicating portfolio was assumed to be given and the analysis only focused on the multi-period valuation of the liability cash flow. Criteria for selection of a replicating portfolio were not analysed. A large part of the current paper focuses on presenting properties of criteria for selection of the replicating portfolio. The criterion advocated as most natural in this paper, in Sect. 2.3, says that a good replicating portfolio is one that makes the need for large equity capital in the reference undertaking small throughout the run-off of the liability. Moreover, in the current paper the value of the liability, see Definitions 2.3 and 2.4 , is implied by no-arbitrage pricing of a derivative security with optionality written on the cumulative cash flow to the owner of the reference undertaking. We demonstrate, in Remark 2.13, that there is a correspondence between the choice of pricing measure used for pricing the derivative security and an adapted process of cost-of-capital rates that defines the capital providers' acceptability criteria for providing solvency capital throughout the run-off of the liability. In [10], all models were specified with respect to the real-world probability measure including the unspecified process of cost-of-capital rates defining the capital provider's acceptability criterion. In contrast, valuation of the owner's option to default given a pricing measure is an essential part of the current paper that is unrelated to the analysis in [10].

Replicating portfolio theory for capital requirement calculation has attracted much interest in recent years. There, the value of a liability cash flow at a future time is modelled as a conditional expected value, with respect to the market's pricing measure, of the sum of discounted future liability cash flows. Since computation of this liability value is typically not feasible, one seeks an accurate approximation by replacing the liability cash flow (or its value) by that of a portfolio of traded replication instruments. Then a risk measure is applied to the approximation of the liability value, yielding an approximation of the capital requirement. In Cambou and Filipović [4] and the works [20-22] of Natolski and Werner, various aspects of this replicating portfolio approach to capital calculations are studied. A fact that somewhat complicates the analysis is that risk measures defining capital requirements are defined with respect 
to the real-world probability measure $\mathbb{P}$, whereas the replication criteria are usually expressed in terms of the market's pricing measure $\mathbb{Q}$. Comparisons of properties and effects of different replication criteria are presented in [20-22]. In [4], it is shown how replicating portfolio theory can be formulated in order to allow efficient replication of liability values exhibiting path-dependence.

Dynamic risk measures and dynamic risk-adjusted values have been analysed in great detail during the last decade; see e.g. Detlefsen and Scandolo [9], Cheridito et al. [8], Artzner et al. [1], Bion-Nadal [3], Cheridito and Kupper [6, 7] and the references therein for important contributions. Much of the research in this area has been aimed at establishing properties and representation results for dynamic risk measures in general functional-analytic settings, particularly for bounded stochastic processes and under convexity requirements for the risk measures. We want to allow models for unbounded liability cash flows. Moreover, limited liability for the owner of the reference undertaking in our setting implies that the dynamic valuation mappings appearing here will in general be nonlinear, nonconvex and nonconcave regardless of any additional structure imposed on the conditional risk measures defining the capital requirements. We only assume very basic properties of the conditional risk measures defining capital requirements, namely so-called translation invariance, monotonicity and normalisation. In particular, these weaker requirements allow conditional versions of the risk measure value-at-risk that is extensively used in practice. Structural results for the selection of replicating portfolios are enabled by assuming that the conditional risk measures also satisfy so-called positive homogeneity; see Theorems 2.28 and 2.30 .

Another approach to market-consistent liability valuation is presented by Pelsser and Stadje [23], combining no-arbitrage valuation and actuarial valuation into a general framework. The authors introduce the notion of a two-step market valuation. First, actuarial pricing of the residual risk remaining after conditioning on the future development of prices of traded assets is done. Second, noticing that the outcome of the first step is a random variable expressed in terms of prices of traded assets, the classical linear financial pricing operator is applied to the outcome of the first step. Further, it is shown in [23] how the two-step market valuation can be extended to a dynamic time-consistent valuation. Our approach to valuation is a two-step marketconsistent valuation in a different sense, based on a hypothetical transfer of a replicating portfolio and the liability to a reference undertaking subject to repeated capital requirements. First, a replicating portfolio is chosen. Then the liability is valued by applying a linear pricing operator to the cumulative cash flow that the policyholders will receive, taking capital requirements and the option to default of the owner of the reference undertaking into account. The economic value of the option to default and its effect on the value of an insurance liability is well known by insurers; see e.g. Hancock et al. [14] and Remark 2.12 in Sect. 2 below. It should be emphasised that in our setting, the pricing measure is just one out of infinitely many that correctly prices traded financial instruments in an incomplete arbitrage-free financial market. In particular, the flexibility to choose the pricing operator allows it to be chosen so that the pricing of nonreplicable insurance risk can be interpreted as a cost-of-capital valuation with risk-averse capital providers; see Remark 2.13 in Sect. 2 for details. 
In Schweizer [24] and Malamud et al. [18], two general multi-period frameworks for the pricing of insurance liabilities are developed. The approaches rely on utility indifference pricing and optimal trading in the financial market.

Motivated by the regulatory framework of Solvency II, and in a multi-period incomplete-market setting, best-estimate reserves are studied in depth in Happ et al. [15] as part of valuing a liability as the sum of a best estimate and a risk margin. The best-estimate reserves are obtained by sequential local risk minimisation as defined in Černý and Kallsen [5]. The value of a liability in our setting can be expressed as the sum of the market price of the replicating portfolio and a term depending on the residual (after replication) liability cash flow; see Definition 2.4. However, the best-estimate reserve is not an object that appears naturally in the present paper.

Approaches to market-consistent valuation based on multi-period mean-variance hedging of insurance liabilities corresponding to a cash flow at a terminal time $T$ are presented in Tsanakas et al. [27] and Barigou and Dhaene [2]. In [27], the hedging instruments are derivatives with binary payoff written on the claims development results, and the liability value is defined as the optimal initial endowment corresponding to the optimal self-financing trading strategy. In [2], the value of the liability is defined as the sum of the financial market price of the mean-variance hedge and a so-called actuarial value of the terminal hedging error. The valuation approaches in both [27] and [2] build upon results found in Schweizer [24, 26], Heath et al. [16] and Černý and Kallsen [5] for mean-variance hedging in a general setting.

Repeated capital requirements together with the option to default form the basis for valuation of liability cash flows in the present paper. Since the option to default does not appear in the approaches to valuation of liabilities in [24, 18, 27, 15, 2], these approaches are quite different from the one in the present paper.

Grosen and Jørgensen [13] consider a derivatives pricing approach to marketconsistent valuation of participating life insurance liabilities in a Black-Scholes model where policyholders are guaranteed a certain payoff at a fixed maturity date. An important feature of the model is that default occurs if the asset value of the insurance company prior to maturity falls below a regulatory boundary corresponding to a fraction of the policyholders' initial deposit compounded with a guaranteed interest rate. Hieber et al. [17] consider a derivatives pricing approach to market-consistent valuation of heterogeneous participating life insurance portfolios with return guarantees. The approach in [17] is similar in spirit to that in [13], but considers general asset-value dynamics for the reference portfolio and possible default at integer times defined as the occurrence of the event that the value of the reference portfolio (asset value) is less than the total policyholder account value (liability value). The run-off scenario in [17, Sect. 3.2] gives a valuation framework that is on the one hand conceptually similar to the one in the present paper, but on the other hand rather different. An essential difference is that in the present paper, externally imposed repeated capital requirements are key drivers of the dynamics of asset and liability values and of occurrence of default.

The paper is organised as follows. The general liability valuation framework is presented in Sect. 2. The three main ingredients are as follows: (1) the value of ownership of the reference undertaking is defined, consistently with classical financial arbitrage valuation, as the no-arbitrage value of the optimally stopped (discounted) 
net cash flow to the owner of the reference undertaking; (2) the value of the liability is defined as the no-arbitrage value of the (discounted) cash flow to the policyholders, stopped optimally from the perspective of the owner of the reference undertaking; and (3) these definitions are shown to be equivalent to two coupled backward recursions for the two values in (1) and (2), and the optimal stopping times are determined explicitly. Sections 2.2 and 2.3 make the general framework operational, in Sect. 2.2 by linking the capital requirements to the liability cash flow in terms of conditional monetary risk measures, and in Sect. 2.3 by presenting criteria for optimal (in various senses) selection of the replicating portfolio. Applying the valuation framework leads to numerical challenges similar to those appearing when valuing American-type financial derivatives. In particular, closed-form solutions are rare exceptions. In Sect. 3, it is shown that under Gaussian model assumptions for the dynamics under both $\mathbb{P}$ and $\mathbb{Q}$, everything can be computed explicitly. Conclusions are found in Sect. 4, and all proofs are found in Sect. 5.

\section{The valuation framework}

We consider time periods $1, \ldots, T$, corresponding time points $0,1, \ldots, T$, and a filtered probability space $(\Omega, \mathcal{F}, \mathbb{F}, \mathbb{P})$, where the filtration is $\mathbb{F}=\left(\mathcal{F}_{t}\right)_{t=0}^{T}$ with $\{\emptyset, \Omega\}=\mathcal{F}_{0} \subseteq \cdots \subseteq \mathcal{F}_{T}=\mathcal{F}$ and where $\mathbb{P}$ denotes the real-world measure. We write $L^{p}\left(\mathcal{F}_{t}, \mathbb{P}\right)$ for the normed linear space of $\mathcal{F}_{t}$-measurable random variables $X$ with norm $\mathbb{E}^{\mathbb{P}}\left[|X|^{p}\right]^{1 / p}$. Equalities and inequalities between random variables should be interpreted in the $\mathbb{P}$-almost sure sense. We use the conventions $\sum_{\ell=k}^{k-1}:=0$ and $\inf \emptyset:=+\infty$ for sums over an empty index set and the infimum of an empty set. We use the notation $x^{+}:=\max (0, x)$.

We assume a given numéraire process $\left(N_{t}\right)_{t=0}^{T}$ and that all financial values are discounted by this numéraire. Although the choice of numéraire is irrelevant for the analysis, we take the numéraire to be the bank account numéraire, i.e., $N_{0}=1$ and $N_{t}$ is the amount at time $t$ from rolling forward an initial unit investment in a locally risk-free traded bond, i.e., a sequence of one-period bonds corresponding to a predictable spot-rate process. In particular, by this choice of numéraire, the time value of money does not appear explicitly at any place. By a risk-free cash flow, we mean a nonrandom sequence in units of the numéraire, i.e., the discounted cash flow is deterministic.

We assume that there exists a strictly positive $(\mathbb{P}, \mathbb{F})$-martingale $\left(D_{t}\right)_{t=0}^{T}$ with $\mathbb{E}^{\mathbb{P}}\left[D_{T}\right]=1$ defining the equivalent pricing measure $\mathbb{Q}$ of an arbitrage-free incomplete financial market via $D_{t}=d \mathbb{Q} /\left.d \mathbb{P}\right|_{\mathcal{F}_{t}}$, i.e., for $u>t$ and a sufficiently integrable $\mathcal{F}_{u}$-measurable $Z$,

$$
\mathbb{E}_{t}^{\mathbb{Q}}[Z]=\frac{1}{D_{t}} \mathbb{E}_{t}^{\mathbb{P}}\left[D_{u} Z\right]
$$

where the subscript $t$ in $\mathbb{E}_{t}^{\mathbb{Q}}$ and $\mathbb{E}_{t}^{\mathbb{P}}$ means conditioning on $\mathcal{F}_{t}$.

In Sect. 2.1 below, we present the valuation framework for general capital requirements in terms of an arbitrary discrete-time stochastic process $\left(R_{t}\right)_{t=0}^{T}$ with $R_{T}=0$, where $R_{t} \in L^{1}\left(\mathcal{F}_{t}, \mathbb{Q}\right)$ for all $t$. In Sect. 2.2, we express $R_{t}$ in terms of conditional monetary risk measures, consistently with current regulatory frameworks. 


\subsection{Valuation of liability cash flows under general capital requirements}

We suppose that in the financial market, there exists an insurance company with an aggregate insurance liability corresponding to a liability cash flow given by the $\mathbb{F}$-adapted stochastic process $X^{o}=\left(X_{t}^{o}\right)_{t=1}^{T}$, the original liability cash flow. Regulation forces the insurance company to comply with externally imposed capital requirements. The requirements put restrictions on the asset portfolio of the insurance company. A set of traded assets is used to form a replicating portfolio intended to offset, to some extent, the liability cash flow. The cash flow of the replicating portfolio is given by an $\mathbb{F}$-adapted stochastic process $X^{r}=\left(X_{t}^{r}\right)_{t=1}^{T}$. Depending on the degree of replicability of the liability cash flow, the replicating portfolio could be simply a long position in the numéraire asset that is turned into payments over time, but also the cash flow from a more sophisticated dynamic hedging strategy. For the latter, we may consider (pre-)strategies in the sense of Heath et al. [16, Definition 2.1] or Schweizer [25, Definition 1.1], taking into account that the hedging strategy aims to hedge an entire cash flow process and not just a payoff at a fixed terminal time. The choice of hedging strategy may be determined by e.g. local risk minimisation or mean-variance hedging. We refer to [16] and [25] for further details and comparisons.

For realistic applications of the valuation framework considered here, it is reasonable to put further restrictions on the set of allowed replicating portfolios. We return to criteria for selection of the replicating portfolio in Sect. 2.3.

The value of the replicating portfolio may be expressed as $\sum_{t=1}^{T} \mathbb{E}_{0}^{\mathbb{Q}}\left[X_{t}^{r}\right]$. However, the liability cash flow cannot be valued as $\sum_{t=1}^{T} \mathbb{E}_{0}^{\mathbb{Q}}\left[X_{t}^{o}\right]$ since $X^{o}$ is the cash flow the policyholders are entitled to, but not necessarily (due to the option to default held by the owner of the reference undertaking) the cash flow they will receive. We define, in accordance with current solvency regulation (Möhr [19] and prescribed by EIOPA, see [11, Article 38]), the value of the liability cash flow $X^{o}$ by considering a hypothetical transfer of the liability and the replicating portfolio to a separate entity referred to as a reference undertaking. The reference undertaking has initially neither assets nor liabilities, and its sole purpose is to manage the run-off of the liability. Ownership of the reference undertaking is achieved by buying it from the insurance company which has transferred its liabilities together with the replicating portfolio and a position $R_{0}$ in the numéraire asset to the reference undertaking. This $R_{0}$ is an amount that makes the reference undertaking meet the imposed capital requirements. Classical arbitrage pricing arguments will determine the price $C_{0}$, specified below, for ownership of the reference undertaking. The benefit of ownership is the right to receive certain dividends, defined below, until either the run-off of the liability cash flow is complete or until letting the reference undertaking default on its obligations to the policyholders. The term default means termination of ownership of the reference undertaking. The precise details are as follows, and illustrated in Fig. 1 below.

- At time $t=0$, the liabilities corresponding to the cash flow $X^{o}$, the replicating portfolio corresponding to the cash flow $X^{r}$ and an amount $R_{0}$ in the numéraire are transferred from the insurance company to the reference undertaking, where $R_{0}$ is the amount making the reference undertaking precisely meet the externally imposed capital requirement. 
- At time $t=0$, by paying the amount $C_{0}$ to the original insurance company, the owner receives full ownership of the reference undertaking. Consequently, the net value of assets transferred from the original insurance company along with the liability is

$$
\mathbb{E}_{0}^{\mathbb{Q}}\left[\sum_{t=1}^{T} X_{t}^{r}\right]+R_{0}-C_{0}
$$

Therefore, in effect, there is no net transfer of capital between the original insurance company and the owner of the reference undertaking. The above is seen to be equivalent to the original insurance company transferring the liabilities, the replicating portfolio and $R_{0}-C_{0}$ to the reference undertaking, followed by the owner of the reference undertaking injecting $C_{0}$ in the reference undertaking-a requirement for the ownership.

- At time $t=1$, the owner has the option to either default on his obligations to the policyholders or not to default.

The decision to default means to give up ownership and transfer $R_{0}$ and the replicating portfolio to the policyholders. The owner neither receives any dividend payment nor incurs any loss upon a decision to default.

If $T>1$ and given the decision not to default, a new amount $R_{1}$ in the numéraire asset is needed to make the reference undertaking precisely meet the externally imposed capital requirement at time 1 . If $R_{0}-R_{1}-X_{1}^{o}+X_{1}^{r} \geq 0$, then the positive surplus $R_{0}-R_{1}-X_{1}^{o}+X_{1}^{r} \geq 0$ is paid to the owner and $X_{1}^{o}$, which the policyholders are entitled to, is paid to the policyholders. If $R_{0}-R_{1}-X_{1}^{o}+X_{1}^{r}<0$, then the owner faces a deficit that must be offset by injecting $-R_{0}+R_{1}+X_{1}^{o}-X_{1}^{r}>0$. Also in this case, $X_{1}^{o}$ is paid to the policyholders.

If $T=1$, then the above description of cash flows to policyholders and owner applies upon setting $R_{1}=0$.

- At time $t \in\{2, \ldots, T\}$, if the owner has not defaulted on his obligations, then the situation is completely analogous to that at time $t=1$ described above.

From the above follows that the owner of the reference undertaking has to decide on a decision rule defining under which circumstances default occurs. The default time is a stopping time $\tau \in \mathcal{S}_{1, T+1}$, where $\mathcal{S}_{t, T+1}$ denotes the set of $\mathbb{F}$-stopping times taking values in $\{t, \ldots, T+1\}$. The event $\{\tau=T+1\}$ is to be interpreted as a complete liability run-off without default at any time.

The cumulative cash flow to the owner can be written as

$$
\sum_{t=1}^{\tau-1}\left(R_{t-1}-R_{t}-X_{t}\right), \quad \text { with } X_{t}:=X_{t}^{o}-X_{t}^{r} .
$$

The value of this cash flow (2.1) is, according to standard arbitrage theory,

$$
\mathbb{E}_{0}^{\mathbb{Q}}\left[\sum_{t=1}^{\tau-1}\left(R_{t-1}-R_{t}-X_{t}\right)\right]
$$


We assume that the owner of the reference undertaking chooses a default time $\tau$ maximising (2.2). Consequently, the value at time 0 of the reference undertaking is

$$
\sup _{\tau \in \mathcal{S}_{1, T+1}} \mathbb{E}_{0}^{\mathbb{Q}}\left[\sum_{t=1}^{\tau-1}\left(R_{t-1}-R_{t}-X_{t}\right)\right] .
$$

For $t \in\{1, \ldots, T\}$, the (discounted) value of the reference undertaking at time $t$, given no default at times $\leq t$, is given by the completely analogous expression upon replacing sup in (2.3) by the essential supremum ess sup with respect to $\mathbb{Q}$ (see Föllmer and Schied [12, Appendix A.5] for details) and conditioning on $\mathcal{F}_{t}$ rather than $\mathcal{F}_{0}$. Notice that since no cash flows occur at times $>T$, the value of the reference undertaking is zero at time $T$. The value of the reference undertaking can thus be identified as the value of an American-type derivative. Details on arbitrage-free pricing of American derivatives can be found in [12, Sect. 6.3].

Definition 2.1 Consider sequences $\left(X_{t}\right)_{t=1}^{T}$ and $\left(R_{t}\right)_{t=0}^{T}$ with $X_{t} \in L^{1}\left(\mathcal{F}_{t}, \mathbb{Q}\right)$ for $t \in\{1, \ldots, T\}, R_{T}=0$ and $R_{t} \in L^{1}\left(\mathcal{F}_{t}, \mathbb{Q}\right)$ for $t \in\{0, \ldots, T-1\}$. Define

$$
\begin{aligned}
C_{t} & :=\underset{\tau \in \mathcal{S}_{t+1, T+1}}{\operatorname{ess} \sup } \mathbb{E}_{t}^{\mathbb{Q}}\left[\sum_{s=t+1}^{\tau-1}\left(R_{s-1}-R_{s}-X_{s}\right)\right], \quad t \in\{0, \ldots, T-1\}, \\
C_{T} & :=0 .
\end{aligned}
$$

We call $C_{t}$ the value of the reference undertaking at time $t$ given no default at times $\leq t$.

Example 2.2 It may be instructive to consider the owner's option to default in the simple one-period setting corresponding to $T=1$. The decision to default at time $t=1$ means that the policyholders receive the cash flow $R_{0}+X_{1}^{r}$ and that the owner of the reference undertaking neither pays nor receives anything. Given the decision not to default at time $t=1$, the policyholders receive $X_{1}^{o}$ and the owner receives the surplus $R_{0}-X_{1}^{o}+X_{1}^{r}$ if this amount is nonnegative, and otherwise pays $-\left(R_{0}-X_{1}^{o}+X_{1}^{r}\right)$ to offset the deficit. Since the owner has no obligation to pay the policyholders to offset a deficit at time $t=1$, it is clear that the option to default is exercised if and only if $R_{0}-X_{1}^{o}+X_{1}^{r}<0$. Consequently, the value of the reference undertaking at time $t=0$ is $\mathbb{E}_{0}^{\mathbb{Q}}\left[\left(R_{0}-X_{1}^{o}+X_{1}^{r}\right)^{+}\right]$. Notice that

$$
\tau= \begin{cases}1 & \text { if } R_{0}-X_{1}^{o}+X_{1}^{r}<0, \\ 2 & \text { if } R_{0}-X_{1}^{o}+X_{1}^{r} \geq 0\end{cases}
$$

gives

$$
\begin{aligned}
\mathbb{E}_{0}^{\mathbb{Q}}\left[\left(R_{0}-X_{1}^{o}+X_{1}^{r}\right)^{+}\right] & =\mathbb{E}_{0}^{\mathbb{Q}}\left[0 \mathbb{1}_{\{\tau=1\}}+\left(R_{0}-X_{1}^{o}+X_{1}^{r}\right) \mathbb{1}_{\{\tau=2\}}\right] \\
& =\sup _{\tau \in \mathcal{S}_{1,2}} \mathbb{E}_{0}^{\mathbb{Q}}\left[\sum_{t=1}^{\tau-1}\left(R_{t-1}-R_{t}-X_{t}\right)\right]
\end{aligned}
$$

which is seen to coincide with (2.4) when $T=1$ and $t=0$. 
Consider $t=0$ and let $\tau_{0}^{*}$ denote an optimal default time such that the supremum in (2.3) is attained for $\tau=\tau_{0}^{*}$. Then the cumulative cash flow to the policyholders is, with $X:=X^{o}-X^{r}$,

$$
\begin{aligned}
\sum_{t=1}^{\tau_{0}^{*}-1} X_{t}^{o}+\sum_{t=\tau_{0}^{*}}^{T} X_{t}^{r}+R_{\tau_{0}^{*}-1} & =\sum_{t=1}^{T} X_{t}^{r}+\sum_{t=1}^{\tau_{0}^{*}-1} X_{t}+R_{\tau_{0}^{*}-1} \\
& =\sum_{t=1}^{T} X_{t}^{r}+R_{0}-\sum_{t=1}^{\tau_{0}^{*}-1}\left(R_{t-1}-R_{t}-X_{t}\right) .
\end{aligned}
$$

Therefore, the arbitrage-free value of the cash flow to the policyholders, i.e., the value of the original insurance company's liabilities, is

$$
\mathbb{E}_{0}^{\mathbb{Q}}\left[\sum_{t=1}^{T} X_{t}^{r}\right]+\mathbb{E}_{0}^{\mathbb{Q}}\left[R_{0}-\sum_{t=1}^{\tau_{0}^{*}-1}\left(R_{t-1}-R_{t}-X_{t}\right)\right],
$$

where the first term is the market price of the replicating portfolio and the second term will be referred to as the residual liability value. For $t \in\{0, \ldots, T\}$, the residual liability value at time $t$ given no default at times $\leq t$ is given by the completely analogous expression upon replacing $\tau_{0}^{*}$ by a default time $\tau_{t}^{*}$ that is optimal as seen from time $t$, i.e.,

$$
R_{t}-\mathbb{E}^{\mathbb{Q}}\left[\sum_{s=t+1}^{\tau_{t}^{*}-1}\left(R_{s-1}-R_{s}-X_{s}\right)\right]=R_{t}-C_{t} .
$$

Notice that

$$
\begin{aligned}
R_{t}-C_{t} & =R_{t}-\underset{\tau \in \mathcal{S}_{t+1, T+1}}{\operatorname{esssup}} \mathbb{E}_{t}^{\mathbb{Q}}\left[\sum_{s=t+1}^{\tau-1}\left(R_{s-1}-R_{s}-X_{s}\right)\right] \\
& =\underset{\tau \in \mathcal{S}_{t+1, T+1}}{\operatorname{essinf}} \mathbb{E}_{t}^{\mathbb{Q}}\left[R_{t}-\sum_{s=t+1}^{\tau-1}\left(R_{s-1}-R_{s}-X_{s}\right)\right] \\
& =\underset{\tau \in \mathcal{S}_{t+1, T+1}}{\operatorname{essinf}} \mathbb{E}_{t}^{\mathbb{Q}}\left[\sum_{s=t+1}^{\tau-1} X_{s}+R_{\tau-1}\right] .
\end{aligned}
$$

Definition 2.3 Consider sequences $\left(X_{t}\right)_{t=1}^{T}$ and $\left(R_{t}\right)_{t=0}^{T}$ with $X_{t} \in L^{1}\left(\mathcal{F}_{t}, \mathbb{Q}\right)$ for $t \in\{1, \ldots, T\}, R_{T}=0$ and $R_{t} \in L^{1}\left(\mathcal{F}_{t}, \mathbb{Q}\right)$ for $t \in\{0, \ldots, T-1\}$. Define

$$
\begin{aligned}
V_{t} & :=\underset{\tau \in \mathcal{S}_{t+1, T+1}}{\operatorname{essinf}} \mathbb{E}_{t}^{\mathbb{Q}}\left[\sum_{s=t+1}^{\tau-1} X_{s}+R_{\tau-1}\right], \quad t \in\{0, \ldots, T-1\}, \\
V_{T} & :=0 .
\end{aligned}
$$

We call $V_{t}$ the value of the residual liability at time $t$ given no default at times $\leq t$. 
Definition 2.4 Consider sequences $\left(X_{t}^{o}\right)_{t=1}^{T},\left(X_{t}^{r}\right)_{t=1}^{T},\left(R_{t}\right)_{t=0}^{T}$ with the properties that $X_{t}^{o}, X_{t}^{r} \in L^{1}\left(\mathcal{F}_{t}, \mathbb{Q}\right)$ for $t \in\{1, \ldots, T\}, R_{T}=0$ and $R_{t} \in L^{1}\left(\mathcal{F}_{t}, \mathbb{Q}\right)$ for $t \in\{0, \ldots, T-1\}$. Set $X_{t}:=X_{t}^{o}-X_{t}^{r}$. Then

$$
L_{t}:=\mathbb{E}_{t}^{\mathbb{Q}}\left[\sum_{s=t+1}^{T} X_{s}^{r}\right]+V_{t}, \quad t \in\{0, \ldots, T\},
$$

where $V_{t}$ is given by Definition 2.3. We call $L_{t}$ the liability value at time $t$ given no default at times $\leq t$.

Remark 2.5 Notice that $L_{0}$ equals

$$
\begin{aligned}
L_{0} & =\mathbb{E}_{0}^{\mathbb{Q}}\left[\sum_{t=1}^{T} X_{t}^{r}\right]+V_{0} \\
& =\mathbb{E}_{0}^{\mathbb{Q}}\left[\sum_{t=1}^{T} X_{t}^{r}\right]+\mathbb{E}_{0}^{\mathbb{Q}}\left[\sum_{t=1}^{\tau_{0}^{*}-1}\left(X_{t}^{o}-X_{t}^{r}\right)+R_{\tau_{0}^{*}-1}\right] \\
& =\mathbb{E}_{0}^{\mathbb{Q}}\left[\sum_{t=1}^{T} X_{t}^{o}\right]+\mathbb{E}_{0}^{\mathbb{Q}}\left[\sum_{t=\tau_{0}^{*}}^{T}\left(X_{t}^{r}-X_{t}^{o}\right)\right]+\mathbb{E}_{0}^{\mathbb{Q}}\left[R_{\tau_{0}^{*}-1}\right],
\end{aligned}
$$

i.e., the sum of the $\mathbb{Q}$-expectations of the original liability cash flow, of the difference from default time onwards between cash flows from the replicating portfolio and original liability, and of the risk capital available at the default time.

Example 2.6 As a continuation of Example 2.2, it may be instructive to consider the cash flow to the policyholders in the simple one-period setting corresponding to $T=1$. As demonstrated in Example 2.2, optimal exercise of the owner's option to default implies that at time $t=1$, the policyholders receive

$$
\left(R_{0}+X_{1}^{r}\right) \mathbb{1}_{\left\{R_{0}-X_{1}^{o}+X_{1}^{r}<0\right\}}+X_{1}^{o} \mathbb{1}_{\left\{R_{0}-X_{1}^{o}+X_{1}^{r} \geq 0\right\}}=X_{1}^{r}+R_{0}-\left(R_{0}-X_{1}^{o}+X_{1}^{r}\right)^{+},
$$

where the first term on the left-hand side is the cash flow to the policyholders upon default and the second is the no-default cash flow. Consequently, the value of the liability, i.e., the $\mathbb{Q}$-expectation of the cash flow to the policyholders, is

$$
\mathbb{E}_{0}^{\mathbb{Q}}\left[X_{1}^{r}+R_{0}-\left(R_{0}-X_{1}^{o}+X_{1}^{r}\right)^{+}\right]=\mathbb{E}_{0}^{\mathbb{Q}}\left[X_{1}^{r}\right]+V_{0}
$$

which is seen to coincide with (2.6) when $T=1$ and $t=0$. Notice that if the owner of the reference undertaking had unlimited liability, i.e., no option to default, then the value of the liability would be

$$
\mathbb{E}_{0}^{\mathbb{Q}}\left[X_{1}^{r}+R_{0}-\left(R_{0}-X_{1}^{o}+X_{1}^{r}\right)\right]=\mathbb{E}_{0}^{\mathbb{Q}}\left[X_{1}^{o}\right],
$$

i.e., the $\mathbb{Q}$-expectation of the cash flow the policyholders are entitled to and also, due to unlimited liability, the cash flow they will receive. 
Remark 2.7 A necessary requirement in order to say that the valuation approach is market-consistent is the requirement that replicable financial liabilities should be valued by their market prices. If $X^{o}$ is fully replicable and the replicating portfolio is chosen so that $X^{r}=X^{o}$, then the residual liability cash flow is $X:=X^{o}-X^{r}=0$. If the capital requirements are such that $X=0$ implies $R_{t}=0$ for all $t$, then $V_{0}=0$ follows from Definition 2.3. This property holds by choosing, as in Sect. 2.2 below, $R_{t}:=\rho_{t}\left(-X_{t+1}-V_{t+1}\right)$ for all $t$, where $\rho_{t}$ is a conditional monetary risk measure in the sense of Definition 2.14. Consequently,

$$
L_{0}:=\mathbb{E}_{0}^{\mathbb{Q}}\left[\sum_{t=1}^{T} X_{t}^{r}\right]+V_{0}=\mathbb{E}_{0}^{\mathbb{Q}}\left[\sum_{t=1}^{T} X_{t}^{o}\right]
$$

is simply the market price of the replicable cash flow $X^{o}$. Hence, market consistency requires that the valuation framework is combined with an appropriate criterion for selection of the replicating portfolio. The criterion for selecting the replicating portfolio does not enter in the mathematics of the general valuation framework considered up to this point. However, the choice of criterion is important to ensure good economic properties and make the framework fully operational. It is necessary that the insurance regulator clearly prescribes the criterion that must be applied in order to obtain unique liability values. Suitable criteria for replicating portfolio selection and further details are presented in Sect. 2.3. See also Remark 2.25 in Sect. 2.3 for further comments on market consistency.

We are now ready to state a key result. It says that the sequences $\left(C_{t}\right)_{t=0}^{T}$ and $\left(V_{t}\right)_{t=0}^{T}$ of values of the reference undertaking and residual liability, respectively, defined in Definitions 2.1 and 2.3, can equivalently be defined as solutions to a pair of backward recursions. Moreover, it provides an explicit expression for the stopping times that are optimal from the perspective of the owner of the reference undertaking.

Theorem 2.8 Consider sequences $\left(X_{t}^{o}\right)_{t=1}^{T},\left(X_{t}^{r}\right)_{t=1}^{T},\left(R_{t}\right)_{t=0}^{T}$ with the properties that $X_{t}^{o}, X_{t}^{r} \in L^{1}\left(\mathcal{F}_{t}, \mathbb{Q}\right)$ for $t \in\{1, \ldots, T\}, R_{T}=0$ and $R_{t} \in L^{1}\left(\mathcal{F}_{t}, \mathbb{Q}\right)$ for $t \in\{0, \ldots, T-1\}$. Set $X_{t}:=X_{t}^{o}-X_{t}^{r}$.

(i) If the sequences $\left(C_{t}\right)_{t=0}^{T}$ and $\left(V_{t}\right)_{t=0}^{T}$ are given by Definitions 2.1 and 2.3 , then

$$
\begin{aligned}
C_{t} & =\mathbb{E}_{t}^{\mathbb{Q}}\left[\left(R_{t}-X_{t+1}-V_{t+1}\right)^{+}\right], \quad C_{T}=0, \\
V_{t} & =R_{t}-\mathbb{E}_{t}^{\mathbb{Q}}\left[\left(R_{t}-X_{t+1}-V_{t+1}\right)^{+}\right], \quad V_{T}=0 .
\end{aligned}
$$

(ii) The stopping times $\left(\tau_{t}^{*}\right)_{t=0}^{T-1}$ given by

$$
\tau_{t}^{*}=\inf \left\{s \in\{t+1, \ldots, T\}: R_{s-1}-X_{s}-V_{s}<0\right\} \wedge(T+1)
$$

are optimal in (2.4) and (2.5).

(iii) If the sequences $\left(C_{t}\right)_{t=0}^{T}$ and $\left(V_{t}\right)_{t=0}^{T}$ are given by (2.7) and (2.8), then for $t \in\{0, \ldots, T-1\}, C_{t}$ and $V_{t}$ are given by (2.4) and (2.5). 
Fig. 1 Illustration of the valuation procedure. At time $t$, the required capital

$R_{t}=V_{t}+C_{t}$ is available. The upper and middle plots show developments to time $t+1$ not leading to default at time $t+1$ since $X_{t+1}+V_{t+1}<R_{t}$. In the upper plot, there is a surplus $R_{t}-R_{t+1}-X_{t+1}>0$, giving a dividend payment at time $t+1$, whereas in the middle plot, a capital injection is needed to ensure solvency. The lower plot illustrates a default event at time $t+1$ since $X_{t+1}+V_{t+1}>R_{t}$
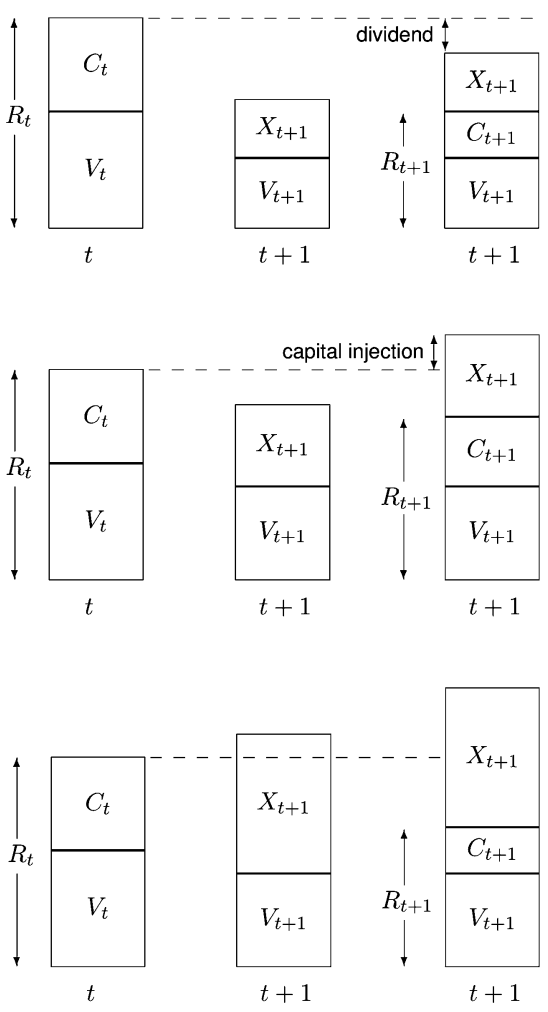

Example 2.9 At time $t$, given that the owner has not exercised the option to default, the balance sheet of the reference undertaking is as follows: the value of the assets is $A_{t}:=\mathbb{E}_{t}^{\mathbb{Q}}\left[\sum_{s=t+1}^{T} X_{s}^{r}\right]+R_{t}$, the value of the liability to policyholders is $L_{t}:=\mathbb{E}_{t}^{\mathbb{Q}}\left[\sum_{s=t+1}^{T} X_{s}^{r}\right]+V_{t}$, and the value of the liability to the owner of the reference undertaking (equity) is $A_{t}-L_{t}=R_{t}-V_{t}=C_{t}$. Figure 1 shows possible evolutions from time $t$ to $t+1$. Just before time $t+1$, the value of the assets is $X_{t+1}^{r}+\mathbb{E}_{t+1}^{\mathbb{Q}}\left[\sum_{s=t+2}^{T} X_{s}^{r}\right]+R_{t}$ and the value of the liability to policyholders is $X_{t+1}^{o}+\mathbb{E}_{t+1}^{\mathbb{Q}}\left[\sum_{s=t+2}^{T} X_{s}^{r}\right]+V_{t+1}$. If the difference (the net asset value) at that time is nonnegative, $R_{t}-X_{t+1}-V_{t+1} \geq 0$, then a rational owner will not exercise the option to default. In this case, at time $t+1$, a positive surplus $R_{t}-R_{t+1}-X_{t+1}>0$ is paid as a dividend to the owner, and a negative surplus $R_{t}-R_{t+1}-X_{t+1}<0$ must be offset by the owner by making a capital injection.

Remark 2.10 The characterisation of the optimal stopping time in Theorem 2.8(ii) says that it is optimal from the owner's perspective to stop (exercise the option to default) at the first time $t$ when the risk capital $R_{t-1}$ rolled forward to time $t$ and reduced by the residual liability cash flow $X_{t}$ falls below the value $V_{t}$ of the remaining residual liability cash flow (the lower plot in Fig. 1 illustrates a situation where stopping at time $t+1$ is optimal). From the perspective of policyholder protection, we may consider the consequences of nonoptimal early stopping 
at time $t$ when $R_{t-1}-X_{t}-V_{t}>0$. At such a time $t$, the value of the assets transferred to the policyholders would be $A_{t-}:=R_{t-1}+X_{t}^{r}+\mathbb{E}_{t}^{\mathbb{Q}}\left[\sum_{s=t+1}^{T} X_{s}^{r}\right]$ and the value of the liabilities would be $L_{t-}:=X_{t}^{o}+\mathbb{E}_{t}^{\mathbb{Q}}\left[\sum_{s=t+1}^{T} X_{s}^{r}\right]+V_{t}$. In particular, $A_{t-}-L_{t-}=R_{t-1}-X_{t}-V_{t}>0$ so the value of the assets transferred to policyholders would exceed the value of the liabilities towards policyholders.

The following remark clarifies how Theorem 2.8 leads to a procedure for sequentially determining the residual liability values $\left(V_{t}\right)_{t=0}^{T}$.

Remark 2.11 We choose in the sequel $R_{t}:=\rho_{t}\left(-X_{t+1}-V_{t+1}\right)$ for conditional monetary risk measures $\rho_{t}$ such as value-at-risk $\operatorname{VaR}_{t, u}$ or expected shortfall $\mathrm{ES}_{t, u}$ that are introduced below in Sect. 2.2. Given that $\left(X_{t}\right)_{t=1}^{T}$ and $\left(\rho_{t}\right)_{t=0}^{T-1}$ are chosen so that $R_{t} \in L^{1}\left(\mathcal{F}_{t}, \mathbb{Q}\right)$, the definition of $\left(V_{t}\right)_{t=0}^{T}$ in Definition 2.3 together with the optimal stopping times in Theorem 2.8(ii) leads to the following procedure for sequentially determining $\left(V_{t}\right)_{t=0}^{T}$ :

- $t=T: R_{T}=0$ and $V_{T}=0$.

$-t=T-1, \ldots, 0: R_{t}=\rho_{t}\left(-X_{t+1}-V_{t+1}\right)$,

$$
\begin{aligned}
\tau_{t}^{*} & =\inf \left\{s \in\{t+1, \ldots, T\}: R_{s-1}-X_{s}-V_{s}<0\right\} \wedge(T+1), \\
V_{t} & =\mathbb{E}_{t}^{\mathbb{Q}}\left[\sum_{s=t+1}^{\tau_{t}^{*}-1} X_{s}+R_{\tau_{t}^{*}-1}\right] .
\end{aligned}
$$

The following remark shows that due to limited liability for the owner of the reference undertaking, the cumulative residual value process is a $(\mathbb{Q}, \mathbb{F})$-submartingale, which further leads to an upper bound on the value of the original liability.

Remark 2.12 Notice from (2.8) that the cumulative residual liability value process

$$
V_{t}^{S}:=\sum_{s=1}^{t} X_{s}+V_{t}, \quad t=0, \ldots, T
$$

is a $(\mathbb{Q}, \mathbb{F})$-submartingale; indeed,

$$
\begin{aligned}
\mathbb{E}_{t}^{\mathbb{Q}}\left[V_{t+1}^{S}\right] & =\sum_{s=1}^{t} X_{s}+R_{t}-\mathbb{E}_{t}^{\mathbb{Q}}\left[R_{t}-X_{t+1}-V_{t+1}\right] \\
& \geq \sum_{s=1}^{t} X_{s}+R_{t}-\mathbb{E}_{t}^{\mathbb{Q}}\left[\left(R_{t}-X_{t+1}-V_{t+1}\right)^{+}\right] \\
& =V_{t}^{S} .
\end{aligned}
$$


Equivalently, $V_{t} \leq \mathbb{E}_{t}^{\mathbb{Q}}\left[\sum_{s=t+1}^{T} X_{s}\right]$ for all $t$. In particular, $V_{0} \leq \mathbb{E}_{0}^{\mathbb{Q}}\left[\sum_{t=1}^{T} X_{t}\right]$ and consequently

$$
L_{0}=\mathbb{E}_{0}^{\mathbb{Q}}\left[\sum_{t=1}^{T} X_{t}^{r}\right]+V_{0} \leq \mathbb{E}_{0}^{\mathbb{Q}}\left[\sum_{t=1}^{T} X_{t}^{o}\right],
$$

regardless of the choice of replicating portfolio. In Hancock at al. [14], the nonnegative value $\mathbb{E}_{0}^{\mathbb{Q}}\left[\sum_{t=1}^{T} X_{t}^{o}\right]-L_{0}$ is referred to as the value of the option to default. This value exists due to limit liability which leads to the possibility that the policyholders do not receive the full payment they are entitled to.

The following remark illustrates that our approach to valuation has a natural costof-capital-valuation interpretation.

Remark 2.13 Notice that

$$
\begin{aligned}
V_{t} & =R_{t}-\mathbb{E}_{t}^{\mathbb{Q}}\left[\left(R_{t}-X_{t+1}-V_{t+1}\right)^{+}\right] \\
& =R_{t}-\frac{1}{1+\eta_{t}} \mathbb{E}_{t}^{\mathbb{P}}\left[\left(R_{t}-X_{t+1}-V_{t+1}\right)^{+}\right]
\end{aligned}
$$

upon defining

$$
\eta_{t}:=\frac{\mathbb{E}_{t}^{\mathbb{P}}\left[\left(R_{t}-X_{t+1}-V_{t+1}\right)^{+}\right]}{\mathbb{E}_{t}^{\mathbb{Q}}\left[\left(R_{t}-X_{t+1}-V_{t+1}\right)^{+}\right]}-1 .
$$

A cost-of-capital valuation results from considering the owner's (capital provider's) time- $t$ one-period acceptability criterion

$$
\mathbb{E}_{t}^{\mathbb{P}}\left[\left(R_{t}-X_{t+1}-V_{t+1}\right)^{+}\right]=\left(1+\eta_{t}\right)\left(R_{t}-V_{t}\right)
$$

which corresponds to an expected excess rate of return $\eta_{t}$ for providing capital with value $C_{t}=R_{t}-V_{t}$ at time $t$. Hence, given a pricing measure $\mathbb{Q}$, the market-consistent value of the liability cash flow can always be interpreted as a cost-of-capital value. It is reasonable to choose $\mathbb{Q}$ consistent with cost-of-capital valuation where the capital provider demands a cost-of-capital rate $\eta_{t} \geq 0$ for all $t$. If $V_{t} \geq \mathbb{E}_{t}^{\mathbb{P}}\left[\sum_{s=t+1}^{T} X_{s}\right]$ for all $t$, which is equivalent to saying that $V^{S}$ in (2.9) is a $(\mathbb{P}, \mathbb{F})$-supermartingale, then $\eta_{t} \geq 0$ for all $t$; indeed, if $V^{S}$ is a $(\mathbb{P}, \mathbb{F})$-supermartingale, then

$$
V_{t} \geq \mathbb{E}_{t}^{\mathbb{P}}\left[X_{t+1}+V_{t+1}\right]=R_{t}-\mathbb{E}_{t}^{\mathbb{P}}\left[R_{t}-X_{t+1}-V_{t+1}\right]
$$

which together with the representation (2.10) for $V_{t}$ gives

$$
\eta_{t} \geq \frac{\mathbb{E}_{t}^{\mathbb{P}}\left[\left(R_{t}-X_{t+1}-V_{t+1}\right)^{+}\right]}{\mathbb{E}_{t}^{\mathbb{P}}\left[R_{t}-X_{t+1}-V_{t+1}\right]}-1 \geq 0 .
$$

By Remark 2.12, $V_{t} \leq \mathbb{E}_{t}^{\mathbb{Q}}\left[\sum_{s=t+1}^{T} X_{s}\right]$ for all $t$. Thus, if $V_{t} \geq \mathbb{E}_{t}^{\mathbb{P}}\left[\sum_{s=t+1}^{T} X_{s}\right]$ for all $t$, then $\mathbb{E}_{t}^{\mathbb{Q}}\left[\sum_{s=t+1}^{T} X_{s}\right] \geq \mathbb{E}_{t}^{\mathbb{P}}\left[\sum_{s=t+1}^{T} X_{s}\right]$ for all $t$. A further consequence of 
assuming that $V_{t} \geq \mathbb{E}_{t}^{\mathbb{P}}\left[\sum_{s=t+1}^{T} X_{s}\right]$ for all $t$ is the following lower bound on the liability value $L_{0}$ :

$$
L_{0}=\mathbb{E}_{0}^{\mathbb{Q}}\left[\sum_{t=1}^{T} X_{t}^{r}\right]+V_{0} \geq \mathbb{E}_{0}^{\mathbb{Q}}\left[\sum_{t=1}^{T} X_{t}^{r}\right]+\mathbb{E}_{0}^{\mathbb{P}}\left[\sum_{t=1}^{T} X_{t}\right] .
$$

\subsection{Capital requirements in terms of conditional monetary risk measures}

The valuation framework presented above is not operational without specifying how the sequence $\left(R_{t}\right)_{t=0}^{T-1}$ depends on the sequences $\left(X_{t}^{o}\right)_{t=1}^{T},\left(X_{t}^{r}\right)_{t=1}^{T}$ and $\left(V_{t}\right)_{t=0}^{T-1}$. In what follows, we define $R_{t}$ in terms of a conditional monetary risk measure $\rho_{t}$ by setting $R_{t}:=\rho_{t}\left(-X_{t+1}-V_{t+1}\right)$.

Definition 2.14 For $p \in[0, \infty]$ and $t \in\{0, \ldots, T-1\}$, a conditional monetary risk measure is a mapping $\rho_{t}: L^{p}\left(\mathcal{F}_{t+1}, \mathbb{P}\right) \rightarrow L^{p}\left(\mathcal{F}_{t}, \mathbb{P}\right)$ satisfying

$$
\begin{aligned}
& \text { if } \lambda \in L^{p}\left(\mathcal{F}_{t}, \mathbb{P}\right) \text { and } Y \in L^{p}\left(\mathcal{F}_{t+1}, \mathbb{P}\right) \text {, then } \rho_{t}(Y+\lambda)=\rho_{t}(Y)-\lambda \text {; } \\
& \text { if } Y, \widetilde{Y} \in L^{p}\left(\mathcal{F}_{t+1}, \mathbb{P}\right) \text { and } Y \leq \widetilde{Y} \text {, then } \rho_{t}(Y) \geq \rho_{t}(\tilde{Y}) \text {; } \\
& \rho_{t}(0)=0 \text {. }
\end{aligned}
$$

Remark 2.15 Notice that the capital requirements are defined in terms of characteristics of $\left(X_{t}\right)_{t=1}^{T}$ and $\left(V_{t}\right)_{t=1}^{T}$ with respect to the probability measure $\mathbb{P}$, whereas the values $V_{t}$ are obtained as solutions to the recursion (2.8) expressed in terms of conditional $\mathbb{Q}$-expectations. This fact give rise to computational challenges. In particular, we need to express the $\mathbb{Q}$-expectations in terms of $\mathbb{P}$-expectations in order to solve the backward recursions in (2.8).

We first recall the conditional monetary risk measures used in current regulations and then consider a slightly more general class of risk measures providing sufficient structure for the subsequent analysis. For $t \geq 0, x \in \mathbb{R}, u \in(0,1)$ and an $\mathcal{F}_{t+1}$-measurable $Z$, let

$$
\begin{aligned}
F_{t,-Z}(x) & :=\mathbb{P}\left[-Z \leq x \mid \mathcal{F}_{t}\right], \\
F_{t,-Z}^{-1}(1-u) & :=\operatorname{essinf}\left\{m \in L^{0}\left(\mathcal{F}_{t}, \mathbb{P}\right): F_{t,-Z}(m) \geq 1-u\right\},
\end{aligned}
$$

and define conditional versions of value-at-risk and expected shortfall as

$$
\operatorname{VaR}_{t, u}(Z):=F_{t,-Z}^{-1}(1-u), \quad \operatorname{ES}_{t, u}(Z):=\frac{1}{u} \int_{0}^{u} \operatorname{VaR}_{t, v}(Z) d v .
$$

$\mathrm{VaR}_{t, u}$ and $\mathrm{ES}_{t, u}$ are special cases of the following more general type of conditional monetary risk measure. Let $M$ be a probability distribution on the Borel subsets of $(0,1)$ such that either $M$ has a bounded density with respect to Lebesgue measure or the support of $M$ is bounded away from 0 and 1 , and let

$$
\rho_{t}(Z):=\int_{0}^{1} F_{t,-Z}^{-1}(u) d M(u) .
$$


Notice that $\operatorname{VaR}_{t, u}$ is obtained by choosing $M$ such that $M(\{1-u\})=1$, and $\mathrm{ES}_{t, u}$ is obtained by choosing $M$ with density $v \mapsto u^{-1} \mathbb{1}_{(1-u, 1)}(v)$.

Theorem 2.16 For $p \in[1, \infty], \rho_{t}$ in (2.12) is a conditional risk measure in the sense of Definition 2.14. In particular, for $p \in[1, \infty], \mathrm{VaR}_{t, u}$ and $\mathrm{ES}_{t, u}$ are conditional monetary risk measures in the sense of Definition 2.14. Moreover, $\rho_{t}(\lambda \cdot)=\lambda \rho_{t}(\cdot)$ for nonnegative constants $\lambda$.

The statement of Theorem 2.16 follows from combining Proposition 4(i) and Remark 5 in Engsner et al. [10]; the proof is therefore omitted.

From (2.7) and (2.8), it follows that $C_{t}$ and $V_{t}$ are determined recursively from $X_{t+1}$ and $V_{t+1}$ via

$$
\begin{aligned}
C_{t}=\gamma_{t}\left(X_{t+1}+V_{t+1}\right), & C_{T}=0, \\
V_{t}=\varphi_{t}\left(X_{t+1}+V_{t+1}\right), & V_{T}=0,
\end{aligned}
$$

where

$$
\begin{aligned}
\gamma_{t}(Y) & :=\mathbb{E}_{t}^{\mathbb{Q}}\left[\left(\rho_{t}(-Y)-Y\right)^{+}\right], \\
\varphi_{t}(Y) & :=\rho_{t}(-Y)-\gamma_{t}(Y) .
\end{aligned}
$$

The following result analyses the mappings $\varphi_{t}$ and how they inherit properties from the conditional monetary risk measures $\rho_{t}$.

Theorem 2.17 (i) Fix $t \in\{0, \ldots, T-1\}$ and $p \in[1, \infty]$. Suppose that we have $D_{t+1} / D_{t} \in L^{\infty}\left(\mathcal{F}_{t+1}, \mathbb{P}\right)$ and that $\rho_{t}$ is a conditional monetary risk measure in the sense of Definition 2.14. Then $\varphi_{t}$ in $(2.14)$ is a mapping from $L^{p}\left(\mathcal{F}_{t+1}, \mathbb{P}\right)$ to $L^{p}\left(\mathcal{F}_{t}, \mathbb{P}\right)$ having the properties

$$
\begin{aligned}
& \text { if } \lambda \in L^{p}\left(\mathcal{F}_{t}, \mathbb{P}\right) \text { and } Y \in L^{p}\left(\mathcal{F}_{t+1}, \mathbb{P}\right) \text {, then } \varphi_{t}(Y+\lambda)=\varphi_{t}(Y)+\lambda \text {; } \\
& \text { if } Y, \widetilde{Y} \in L^{p}\left(\mathcal{F}_{t+1}, \mathbb{P}\right) \text { and } Y \leq \tilde{Y} \text {, then } \varphi_{t}(Y) \leq \varphi_{t}(\tilde{Y}) \text {; } \\
& \varphi_{t}(0)=0 \text {. }
\end{aligned}
$$

(ii) Fix $t \in\{0, \ldots, T-1\}$ and $1 \leq p_{1}<p_{2}$. Suppose $D_{t+1} / D_{t} \in L^{r}\left(\mathcal{F}_{t+1}, \mathbb{P}\right)$ for every $r \geq 1$. Suppose further that for any $p \in\left[p_{1}, p_{2}\right], \rho_{t}$ is a conditional monetary risk measure in the sense of Definition 2.14. Then for any $\varepsilon>0$ such that $p-\varepsilon \geq p_{1}$, $\varphi_{t}$ in (2.14) can be defined as a mapping from $L^{p}\left(\mathcal{F}_{t+1}, \mathbb{P}\right)$ to $L^{p-\varepsilon}\left(\mathcal{F}_{t}, \mathbb{P}\right)$ having the properties (2.15)-(2.17).

Remark 2.18 The value $V_{t}=V_{t}(X)$ may be seen as the result of applying the mapping $V_{t}: L^{p}\left(\left(\mathcal{F}_{t}\right)_{t=1}^{T}, \mathbb{P}\right) \rightarrow L^{p-\varepsilon}\left(\mathcal{F}_{t}, \mathbb{P}\right)$ to $X \in L^{p}\left(\left(\mathcal{F}_{t}\right)_{t=1}^{T}, \mathbb{P}\right)$ for a suitable $\varepsilon \geq 0$ according to Theorem 2.17. If $\left(V_{t}\right)_{t=0}^{T}$ satisfies (2.13), where $\varphi_{t}$ satisfies (2.15)-(2.17), then $\left(V_{t}\right)_{t=0}^{T}$ satisfies the property called time consistency: For every pair of times $(s, t)$ with $s \leq t$, the two conditions $\left(X_{u}\right)_{u=1}^{t}=\left(\widetilde{X}_{u}\right)_{u=1}^{t}$ and $V_{t}(X) \leq V_{t}(\tilde{X})$ together imply $V_{s}(X) \leq V_{s}(\tilde{X})$. Detailed investigations of time consistency and related concepts can be found in e.g. Cheridito and Kupper [6, 7]. 
The requirement $D_{t+1} / D_{t} \in L^{\infty}\left(\mathcal{F}_{t+1}, \mathbb{P}\right)$ in statement (i) of Theorem 2.17 leads to a cleaner definition of the mappings $\gamma_{t}, \varphi_{t}$. However, the boundedness of $D_{t+1} / D_{t}$ may be a too restrictive requirement. Finiteness of all moments of $D_{t+1} / D_{t}$ as in statement (ii) is an appropriate requirement for the subsequent analysis here.

Under the assumptions of Theorem 2.17(i) or (ii), it follows from (2.13) and (2.15) that

$$
V_{t}=\varphi_{t} \circ \cdots \circ \varphi_{T-1}\left(\sum_{s=t+1}^{T} X_{s}\right),
$$

where $\varphi_{t} \circ \cdots \circ \varphi_{T-1}$ denotes the composition of the mappings $\varphi_{t}, \ldots, \varphi_{T-1}$. Definition 2.3 defines the residual liability values $\left(V_{t}\right)_{t=0}^{T-1}$ given the sequences $\left(R_{t}\right)_{t=0}^{T-1}$ and $\left(X_{t}\right)_{t=1}^{T}$ satisfying $R_{t}, X_{t} \in L^{1}\left(\mathcal{F}_{t}, \mathbb{Q}\right)$. However, typically one starts with conditional monetary risk measures $\rho_{t}$ of the kind in (2.12) and an adapted residual liability cash flow defined with respect to $\mathbb{P}$ and not $\mathbb{Q}$. The following result says that this approach to defining the value of the residual liability cash flow and the value of the reference undertaking is fully consistent with Definitions 2.1 and 2.3.

Theorem 2.19 Fix $p>1$ and for all $t \in\{1, \ldots, T\}$, let $X_{t} \in L^{p}\left(\mathcal{F}_{t}, \mathbb{P}\right)$, let $D_{t} \in L^{r}\left(\mathcal{F}_{t}, \mathbb{P}\right)$ for all $r \in[0, \infty)$, and for all $t \in\{0, \ldots, T-1\}$, let $\rho_{t}$ be a conditional monetary risk measure satisfying (2.12). Set $\widetilde{V}_{T}:=0, \widetilde{R}_{T}:=0, \widetilde{C}_{T}:=0$ and for $t \in\{0, \ldots, T-1\}$, set

$$
\begin{aligned}
& \widetilde{V}_{t}:=\varphi_{t} \circ \cdots \circ \varphi_{T-1}\left(\sum_{s=t+1}^{T} X_{s}\right), \\
& \widetilde{R}_{t}:=\rho_{t}\left(-X_{t+1}-\widetilde{V}_{t+1}\right), \\
& \widetilde{C}_{t}:=\gamma_{t}\left(X_{t+1}+\widetilde{V}_{t+1}\right) .
\end{aligned}
$$

Then $X_{t}, \widetilde{R}_{t} \in L^{1}\left(\mathcal{F}_{t}, \mathbb{Q}\right)$ for $t \in\{1, \ldots, T\}$, and for $t \in\{0, \ldots, T-1\}$,

$$
\begin{aligned}
& \widetilde{C}_{t}=\underset{\tau \in \mathcal{S}_{t+1, T+1}}{\operatorname{ess} \sup } \mathbb{E}_{t}^{\mathbb{Q}}\left[\sum_{s=t+1}^{\tau-1}\left(\widetilde{R}_{s-1}-\widetilde{R}_{s}-X_{s}\right)\right], \\
& \widetilde{V}_{t}=\underset{\tau \in \mathcal{S}_{t+1, T+1}}{\operatorname{essinf}} \mathbb{E}_{t}^{\mathbb{Q}}\left[\sum_{s=t+1}^{\tau-1} X_{s}+\widetilde{R}_{\tau-1}\right] .
\end{aligned}
$$

The following result essentially says the following: (1) Adding a nonrandom cash flow to the original insurance company's replicating portfolio will not affect the value $L_{0}$ of the liability cash flow. The reason is that $V_{0}$ will change accordingly to offset the effect of the added nonrandom cash flow. (2) The value of the ownership of the reference undertaking is zero at all times if and only if $\sum_{t=1}^{T} X_{t}=K$ for some constant $K$. (3) If the replicating portfolio superreplicates the original liability cash flow, then the value $L_{0}$ of the liability is not greater than the market price of the replicating portfolio. Similarly, if the replicating portfolio subreplicates the original liability 
cash flow, then the value $L_{0}$ of the liability is not smaller than the market price of the replicating portfolio.

Theorem 2.20 Suppose that $\left(V_{t}\right)_{t=0}^{T}$ satisfies (2.13) with $\left(\varphi_{t}\right)_{t=0}^{T-1}$ given by (2.14) satisfying one of the statements (i) or (ii) of Theorem 2.17.

(i) If $\widetilde{X}^{r}=X^{r}+b$, where $b_{t}$ is $\mathcal{F}_{0}$-measurable for all $t \in\{1, \ldots, T\}$, then with $\tilde{X}:=X^{o}-\tilde{X}^{r}$,

$$
\sum_{t=1}^{T} \mathbb{E}_{0}^{\mathbb{Q}}\left[\tilde{X}_{t}^{r}\right]+V_{0}(\tilde{X})=\sum_{t=1}^{T} \mathbb{E}_{0}^{\mathbb{Q}}\left[X_{t}^{r}\right]+V_{0}(X),
$$

and for all $t \in\{0, \ldots, T-1\}$,

$$
\begin{aligned}
\widetilde{C}_{t} & =\mathbb{E}_{t}^{\mathbb{Q}}\left[\left(\rho_{t}\left(-\tilde{X}_{t+1}-V_{t+1}(\tilde{X})\right)-\tilde{X}_{t+1}-V_{t+1}(\tilde{X})\right)^{+}\right] \\
& =\mathbb{E}_{t}^{\mathbb{Q}}\left[\left(\rho_{t}\left(-X_{t+1}-V_{t+1}(X)\right)-X_{t+1}-V_{t+1}(X)\right)^{+}\right] \\
& =C_{t} .
\end{aligned}
$$

(ii) If there is an $\mathcal{F}_{0}$-measurable $K$ such that $\sum_{t=1}^{T} X_{t}=K$, then $C_{t}=0$ for all $t \in\{0, \ldots, T-1\}$ and $K=V_{0}$.

(iii) If for all $t \in\{0, \ldots, T-1\}, C_{t}=0$ and $\rho_{t}$ has the property

$$
\text { if } Y \in L^{p}\left(\mathcal{F}_{t+1}, \mathbb{P}\right) \text { and } \mathbb{P}_{t}\left[Y \geq \rho_{t}(-Y)\right]=1 \text {, then } Y \in L^{p}\left(\mathcal{F}_{t}, \mathbb{P}\right) \text {, }
$$

then $\sum_{t=1}^{T} X_{t}=V_{0}$.

(iv) If $X_{t}^{r} \geq X_{t}^{o}$ for all $t \in\{1, \ldots, T\}$, then $L_{0} \leq \mathbb{E}_{0}^{\mathbb{Q}}\left[\sum_{t=1}^{T} X_{t}^{r}\right]$.

(v) If $X_{t}^{r} \leq X_{t}^{o}$ for all $t \in\{1, \ldots, T\}$, then $L_{0} \geq \mathbb{E}_{0}^{\mathbb{Q}}\left[\sum_{t=1}^{T} X_{t}^{r}\right]$.

Corollary 2.21 Suppose that $\left(V_{t}\right)_{t=0}^{T}$ satisfies (2.13) with $\left(\varphi_{t}\right)_{t=0}^{T-1}$ given by (2.14) with $\rho_{t}=\mathrm{ES}_{t, p}$ for some $p \in(0,1)$ and all $t$. Then $C_{t}=0$ for all $t \in\{0, \ldots, T-1\}$ if and only if $\sum_{t=1}^{T} X_{t}=V_{0}$.

The following example illustrates the valuation procedure under a strong independence assumption.

Example 2.22 Consider a cash flow $\left(X_{t}\right)_{t=1}^{T}$ such that $X_{t}$ is independent of $\mathcal{F}_{s}$ for $s<t$ and suppose that $\rho_{t}$ is a conditional monetary risk measure satisfying (2.12). Then, since $X_{T}$ is independent of $\mathcal{F}_{T-1}$,

$$
\begin{aligned}
V_{T-1} & =\rho_{T-1}\left(-X_{T}\right)-\mathbb{E}_{T-1}^{\mathbb{Q}}\left[\left(\rho_{T-1}\left(-X_{T}\right)-X_{T}\right)^{+}\right] \\
& =\rho_{0}\left(-X_{T}\right)-\mathbb{E}_{0}^{\mathbb{Q}}\left[\left(\rho_{0}\left(-X_{T}\right)-X_{T}\right)^{+}\right],
\end{aligned}
$$


and since $V_{T-1}$ here is nonrandom and $X_{T-1}$ is independent of $\mathcal{F}_{T-2}$,

$$
\begin{aligned}
V_{T-2}= & \rho_{T-2}\left(-X_{T-1}-V_{T-1}\right) \\
& -\mathbb{E}_{T-2}^{\mathbb{Q}}\left[\left(\rho_{T-2}\left(-X_{T-1}-V_{T-1}\right)-X_{T-1}-V_{T-1}\right)^{+}\right] \\
= & V_{T-1}+\rho_{0}\left(-X_{T-1}\right)-\mathbb{E}_{0}^{\mathbb{Q}}\left[\left(\rho_{0}\left(-X_{T-1}\right)-X_{T-1}\right)^{+}\right] .
\end{aligned}
$$

Repeating the above arguments yields

$$
V_{t}=V_{t+1}+\rho_{0}\left(-X_{t+1}\right)-\mathbb{E}_{0}^{\mathbb{Q}}\left[\left(\rho_{0}\left(-X_{t+1}\right)-X_{t+1}\right)^{+}\right], \quad V_{T}=0,
$$

i.e., $V_{t}=\sum_{s=t+1}^{T}\left(\rho_{0}\left(-X_{s}\right)-\mathbb{E}_{0}^{\mathbb{Q}}\left[\left(\rho_{0}\left(-X_{S}\right)-X_{s}\right)^{+}\right]\right)$is nonrandom for all $t$. In particular,

$$
\begin{aligned}
& R_{t}=\rho_{0}\left(-X_{t+1}-V_{t+1}\right)=\rho_{0}\left(-X_{t+1}\right)+V_{t+1} \\
& C_{t}=\mathbb{E}_{0}^{\mathbb{Q}}\left[\left(\rho_{0}\left(-X_{t+1}-V_{t+1}\right)-X_{t+1}-V_{t+1}\right)^{+}\right]=\mathbb{E}_{0}^{\mathbb{Q}}\left[\left(\rho_{0}\left(-X_{t+1}\right)-X_{t+1}\right)^{+}\right] .
\end{aligned}
$$

If further there exist an i.i.d. sequence $\left(Z_{t}\right)_{t=1}^{T}$ and nonrandom sequences $\left(\mu_{t}\right)_{t=1}^{T}$ and $\left(\sigma_{t}\right)_{t=1}^{T}$, with $\sigma_{t}$ nonnegative, such that $X_{t}$ and $\mu_{t}+\sigma_{t} Z_{t}$ are equal in distribution, then

$$
\begin{aligned}
C_{t} & =\sigma_{t} \mathbb{E}_{0}^{\mathbb{Q}}\left[\left(\rho_{0}\left(-Z_{1}\right)-Z_{1}\right)^{+}\right], \\
V_{t} & =\sum_{s=t+1}^{T}\left(\mu_{s}+\sigma_{s}\left(\rho_{0}\left(-Z_{1}\right)-\mathbb{E}_{0}^{\mathbb{Q}}\left[\left(\rho_{0}\left(-Z_{1}\right)-Z_{1}\right)^{+}\right]\right)\right) .
\end{aligned}
$$

In particular, whereas there is a recursive relation (2.8) relating $V_{t}$ to $V_{t+1}$, there is in general no similar relation relating $C_{t}$ to $C_{t+1}$.

\subsection{Replicating portfolios}

Definition 2.4 defines the initial value $L_{0}$ of the liability cash flow as the sum of the market price $\mathbb{E}_{0}^{\mathbb{Q}}\left[\sum_{t=1}^{T} X_{t}^{r}\right]$ of a replicating portfolio and the value $V_{0}$ of the residual liability cash flow. Alternatively, $L_{0}$ is the $\mathbb{Q}$-expectation of the cash flow to the policyholders, optimally stopped by the owner of the reference undertaking. As stated in Remark 2.7, in order to talk about the value of the liability cash flow, a choice of replicating portfolio must be made. The original insurance company selects a replicating portfolio earmarked for the liability cash flow before an eventual transfer of liabilities to the reference undertaking. It should be emphasised that the cash flow $X^{r}$ of the replicating portfolio may in principle come from an arbitrarily sophisticated dynamic strategy depending on the chosen criterion for replicating portfolio selection and the set of replication instruments. The only hard restriction is that the cash flow $X^{r}$ is a result of a portfolio or strategy decided by the original insurance company in accordance with rules decided by the regulator, and that the owner of the reference undertaking may not in any way influence the outcome of $X^{r}$. Regardless 
of which replicating portfolio is selected, the position $R_{0}$ in the numéraire asset will always be such that the reference undertaking meets the externally imposed capital requirement.

The sole purpose of the reference undertaking is to manage the run-off of the liabilities. From a regulator's perspective, there are no reasons to accept an unnecessary large balance sheet of the reference undertaking due to $C_{0}$ being large as a consequence of poor replication of the liabilities. Recall Example 2.9 that explains the balance sheet of the reference undertaking. Below we recall well-studied criteria for selection of replicating portfolios and present a new criterion, see (2.23), that says that a good replicating portfolio is one that makes it likely that all $C_{t}$ are small. That is, we advocate selecting the replicating portfolio such that the balance sheet of the reference undertaking throughout the run-off period is likely to be small at all times.

Consider $m$ (discounted) cash flows $X^{f, k}=\left(X_{t}^{f, k}\right)_{t=1}^{T}, k=1, \ldots, m$, of available financial instruments and denote by $X^{f}$ the $\mathbb{R}^{m}$-valued process such that $X_{t}^{f}$ denotes the (column) vector of time- $t$ cash flows of the $m$ instruments. A portfolio with portfolio-weight vector $v \in \mathbb{R}^{m}$, representing the number of units of the $m$ instruments, generates the cash flow $v^{\mathrm{T}} X_{t}^{f}$ at time $t$.

Various criteria for selection of replicating portfolio have been considered in the literature. The optimisation problem

$$
\inf _{v \in \mathbb{R}^{m}} \sum_{t=1}^{T} \mathbb{E}_{0}^{\mathbb{Q}}\left[\left(X_{t}^{o}-v^{\mathrm{T}} X_{t}^{f}\right)^{2}\right]^{1 / 2}
$$

is referred to as cash flow matching in Natolski and Werner [21]. Under mild conditions, it is shown in Theorems 1 (and 2) in [21] that an optimal (unique optimal) solution exists. An alternative cash-flow-matching problem is

$$
\inf _{v \in \mathbb{R}^{m}} \sum_{t=1}^{T} \mathbb{E}_{0}^{\mathbb{Q}}\left[\left(X_{t}^{o}-v^{\mathrm{T}} X_{t}^{f}\right)^{2}\right] .
$$

Comparisons between (2.20) and (2.21) are found in Natolski and Werner [20]. The optimisation problem

$$
\inf _{v \in \mathbb{R}^{m}} \mathbb{E}_{0}^{\mathbb{Q}}\left[\left(\sum_{t=1}^{T}\left(X_{t}^{o}-v^{\mathrm{T}} X_{t}^{f}\right)\right)^{2}\right]^{1 / 2}
$$

is referred to as terminal-value matching in [20-22]. It is a standard quadratic optimisation problem with explicit solution

$$
\widehat{v}=\mathbb{E}_{0}^{\mathbb{Q}}\left[\left(\begin{array}{ccc}
X^{f, 1} X^{f, 1} & \ldots & X^{f, 1} X^{f, m} \\
\vdots & & \vdots \\
X^{f, m}, X^{f, 1} & \ldots & X^{f, m} X^{f, m}
\end{array}\right)\right]^{-1} \mathbb{E}_{0}^{\mathbb{Q}}\left[\left(\begin{array}{c}
X_{\cdot}^{o} X^{f, 1} \\
\vdots \\
X_{.}^{o} X^{f, m}
\end{array}\right)\right],
$$

provided that the matrix inverse exists, where the subscript $\cdot$ means summation over the index $t$. 
A replicating portfolio selection criterion should have the property that if perfect replication is possible, then the optimal replicating portfolio cash flow $\widehat{v}^{\mathrm{T}} X^{f}$ satisfies $X^{o}=\widehat{v}^{\mathrm{T}} X^{f}$. This requirement ensures market-consistent liability values, because then $L_{0}=\sum_{t=1}^{T} \mathbb{E}_{0}^{\mathbb{Q}}\left[X_{t}^{o}\right]$ for a replicable liability cash flow.

Remark 2.23 The versions of the optimisation problems (2.20)-(2.22) obtained by replacing the expectation $\mathbb{E}_{0}^{\mathbb{Q}}$ by $\mathbb{E}_{0}^{\mathbb{P}}$ may also be reasonable. Notice that if the only available replication instruments are zero-coupon bonds in the numéraire asset of all maturities $t=1, \ldots, T$ (or, equivalently, European call options on the numéraire asset with maturities $t=1, \ldots, T$ and common strike price 0 ), then $m=T$ and $X^{f}$ is the $T \times T$ identity matrix. In this case,

$$
\inf _{v \in \mathbb{R}^{m}} \sum_{t=1}^{T} \mathbb{E}_{0}^{\mathbb{P}}\left[\left(X_{t}^{o}-v^{\mathrm{T}} X_{t}^{f}\right)^{2}\right]=\inf _{v \in \mathbb{R}^{m}} \sum_{t=1}^{T} \mathbb{E}_{0}^{\mathbb{P}}\left[\left(X_{t}^{o}-v_{t}\right)^{2}\right],
$$

and the unique optimal solution is $\widehat{v}=\mathbb{E}_{0}^{\mathbb{P}}\left[X^{o}\right]$ which is referred to as the actuarial best-estimate reserve.

Notice that given the above set of replication instruments, any $\widehat{v}$ which satisfies $\sum_{t=1}^{T} \widehat{v}_{t}=\sum_{t=1}^{T} \mathbb{E}_{0}^{\mathbb{P}}\left[X_{t}^{o}\right]$ is a solution to the version of the terminal value problem (2.22) obtained by replacing the expectation $\mathbb{E}_{0}^{\mathbb{Q}}$ by $\mathbb{E}_{0}^{\mathbb{P}}$.

In our setting, the value of the reference undertaking at time $t$ is

$$
C_{t}=\mathbb{E}_{t}^{\mathbb{Q}}\left[\left(\rho_{t}\left(-X_{t+1}-V_{t+1}\right)-X_{t+1}-V_{t+1}\right)^{+}\right] .
$$

The amount $C_{t}$ must be provided by the owner of the reference undertaking in order to meet the externally imposed capital requirements. From the above representation of $C_{t}$ together with (2.18), it is seen that a high uncertainty in the outcome of the aggregate residual cash flow (replication error) $\sum_{s=t+1}^{T} X_{s}$ corresponds to a large value of $C_{t}$. It is in the policyholders' interest that the cash flow they are entitled to is likely to be close to the cash flow they will receive. Therefore, the regulator should enforce a selection of replicating portfolios that makes it likely that all the $C_{t}$ are small, i.e., that the values of the reference undertaking throughout the run-off of the liabilities are likely to be uniformly small. Recall from Theorem 2.20 and Corollary 2.21 that under mild conditions, $\sum_{t=1}^{T} X_{t}$ is nonrandom if and only if $C_{t}=0$ for all $t$. We therefore consider the optimisation problem

$$
\inf _{v \in \mathbb{R}^{m}} \psi(v) \quad \text { with } \psi(v):=\mathbb{E}_{0}^{\mathbb{Q}}\left[\max _{t \in\{0, \ldots, T-1\}} C_{t}^{v}\right]
$$

where for $t=0, \ldots, T-1$,

$$
C_{t}^{v}:=\mathbb{E}_{t}^{\mathbb{Q}}\left[\left(R_{t}^{v}-X_{t+1}^{v}-V_{t+1}^{v}\right)^{+}\right]
$$

with $X^{v}:=X^{o}-v^{\mathrm{T}} X^{f}, V_{t}^{v}:=V_{t}\left(X^{v}\right), R_{t}^{v}:=\rho_{t}\left(-X_{t+1}^{v}-V_{t+1}^{v}\right)$.

From Theorem 2.20(i), it follows that $C_{t}^{v}$ for all $t$ and consequently also $\psi(v)$ in (2.23) are invariant under translations of $X^{v}$ by nonrandom sequences (risk-free cash 
flows). Therefore the optimisation problem (2.23) will not have a unique solution if risk-free cash flows are included as replication instruments.

Remark 2.24 If $\left(R_{t}^{S}\right)_{t=0}^{T}$ given by $R_{t}^{S}:=R_{t}+\sum_{s=1}^{t} X_{s}$ is a $(\mathbb{Q}, \mathbb{F})$-supermartingale, then $\left(C_{t}\right)_{t=0}^{T}$ is a $(\mathbb{Q}, \mathbb{F})$-supermartingale; indeed,

$$
\begin{aligned}
C_{t} & =\mathbb{E}_{t}^{\mathbb{Q}}\left[\left(R_{t}-X_{t+1}-R_{t+1}+C_{t+1}\right)^{+}\right] \\
& \geq R_{t}-\mathbb{E}_{t}^{\mathbb{Q}}\left[X_{t+1}+R_{t+1}\right]+\mathbb{E}_{t}^{\mathbb{Q}}\left[C_{t+1}\right] \\
& \geq \mathbb{E}_{t}^{\mathbb{Q}}\left[C_{t+1}\right],
\end{aligned}
$$

and then $C_{0}=0$ implies that $C_{t}=0$ for all $t$. Notice that $\left(R_{t}^{S}\right)_{t=0}^{T}$ is a $(\mathbb{Q}, \mathbb{F})$-supermartingale exactly when $\mathbb{E}_{t}^{\mathbb{Q}}\left[R_{t}-R_{t+1}-X_{t+1}\right] \geq 0$ for all $t$. From this observation and (2.1), it follows that the assumption that $\left(R_{t}^{S}\right)_{t=0}^{T}$ is a $(\mathbb{Q}, \mathbb{F})$-supermartingale means that at every time $t$, the financial value of the next-period cash flow to the owner of the reference undertaking is nonnegative. Finally, it should be noted that it is easy to find examples where $\left(C_{t}\right)_{t=0}^{T}$ is not a $(\mathbb{Q}, \mathbb{F})$-supermartingale; see Example 2.22 above.

The optimisation problems (2.20)-(2.23) can all be expressed as

$$
\inf _{v \in \mathbb{R}^{m}} \Psi\left(X^{o}-v^{\mathrm{T}} X^{f}\right)
$$

for a mapping $\Psi: L^{p}\left(\left(\mathcal{F}_{t}\right)_{t=1}^{T}, \mathbb{Q}\right) \rightarrow \mathbb{R}_{+}$satisfying $\Psi(0)=0$, i.e., optimality of perfect replication. Existence of a minimiser $\widehat{X}^{r}:=\widehat{v}^{\mathrm{T}} X^{f}$ can be expressed as

$$
\Psi\left(X^{o}-\widehat{X}^{r}\right)=\inf _{v \in \mathbb{R}^{m}} \Psi\left(X^{o}-v^{\mathrm{T}} X^{f}\right) .
$$

Conditions for the existence of a minimiser $\widehat{v}$ in (2.23) are presented in Theorem 2.30 below.

Remark 2.25 Consider a liability cash flow $X^{\circ}$ that is not fully replicable and assume the existence of a unique minimiser $\widehat{v}$ to (2.24). Writing $V_{0}=V_{0}\left(X^{o}-X^{r}\right)$ to make explicit the dependence of $V_{0}$ on the residual liability cash flow $X^{o}-X^{r}$, the value of $X^{o}$, optimally replicated, is

$$
L_{0}=\widehat{v}^{\mathrm{T}} \mathbb{E}_{0}^{\mathbb{Q}}\left[\sum_{t=1}^{T} X_{t}^{f}\right]+V_{0}\left(X^{o}-\widehat{v}^{\mathrm{T}} X^{f}\right) .
$$

Consider another liability cash flow $\widetilde{X}^{o}:=X^{o}+Y^{o}$ which only differs from $X^{o}$ by a fully replicable term $Y^{o}=v_{Y}^{\mathrm{T}} X^{f}$. Since

$$
\inf _{v \in \mathbb{R}^{m}} \Psi\left(\tilde{X}^{o}-v^{\mathrm{T}} X^{f}\right)=\inf _{v \in \mathbb{R}^{m}} \Psi\left(\tilde{X}^{o}-\left(v+v_{Y}\right)^{\mathrm{T}} X^{f}\right)=\inf _{v \in \mathbb{R}^{m}} \Psi\left(X^{o}-v^{\mathrm{T}} X^{f}\right),
$$


the optimal replicating portfolio weights are given by $\widehat{v}+v_{Y}$ which implies that the value of $\widetilde{X}^{o}$, optimally replicated, is

$$
\begin{aligned}
\widetilde{L}_{0} & =\left(\widehat{v}+v_{Y}\right)^{\mathrm{T}} \mathbb{E}_{0}^{\mathbb{Q}}\left[\sum_{t=1}^{T} X_{t}^{f}\right]+V_{0}\left(\tilde{X}^{o}-\left(\widehat{v}+v_{Y}\right)^{\mathrm{T}} X^{f}\right) \\
& =\left(\widehat{v}+v_{Y}\right)^{\mathrm{T}} \mathbb{E}_{0}^{\mathbb{Q}}\left[\sum_{t=1}^{T} X_{t}^{f}\right]+V_{0}\left(X^{o}-\widehat{v}^{\mathrm{T}} X^{f}\right) \\
& =\mathbb{E}_{0}^{\mathbb{Q}}\left[\sum_{t=1}^{T} Y_{t}^{o}\right]+L_{0},
\end{aligned}
$$

i.e., the value of $\widetilde{X}^{o}$ differs from that of $X^{o}$ by the market price of $Y^{o}$. This property of $L_{0}$ seen as a valuation operator is essentially market consistency in the sense of Pelsser and Stadje [23, Definition 3.1].

Remark 2.26 The deterministic replicating portfolio cash flow $\widehat{X}^{r}=\mathbb{E}_{0}^{\mathbb{P}}\left[X^{o}\right]$ corresponds to a classical actuarial best-estimate reserve, and solves a cash-flow-matching problem with only risk-free cash flows in the numéraire asset as replication instruments; see Remark 2.23. In this case, by Theorem 2.20,

$$
L_{0}=\sum_{t=1}^{T} \mathbb{E}_{0}^{\mathbb{P}}\left[X_{t}^{o}\right]+V_{0}\left(X^{o}-\mathbb{E}_{0}^{\mathbb{P}}\left[X^{o}\right]\right)=V_{0}\left(X^{o}\right) .
$$

In particular, if $V_{0}\left(X^{o}\right) \geq \sum_{t=1}^{T} \mathbb{E}_{0}^{\mathbb{P}}\left[X_{t}^{o}\right]$, then $L_{0} \geq \sum_{t=1}^{T} \mathbb{E}_{0}^{\mathbb{P}}\left[X_{t}^{o}\right]$. As noted in Remark 2.23, any deterministic cash flow $\widehat{X}^{r}$ with $\sum_{t=1}^{T} \widehat{X}_{t}^{r}=\sum_{t=1}^{T} \mathbb{E}_{0}^{\mathbb{P}}\left[X_{t}^{o}\right]$ is a solution to the (alternative) terminal value problem

$$
\inf _{v \in \mathbb{R}^{m}} \mathbb{E}_{0}^{\mathbb{P}}\left[\left(\sum_{t=1}^{T}\left(X_{t}^{o}-v^{\mathrm{T}} X_{t}^{f}\right)\right)^{2}\right],
$$

with only risk-free cash flows in the numéraire asset as replication instruments. In this case, by Theorem 2.20,

$$
L_{0}=\sum_{t=1}^{T} \mathbb{E}_{0}^{\mathbb{Q}}\left[\widehat{X}_{t}^{r}\right]+V_{0}\left(X^{o}-\widehat{X}^{r}\right)=V_{0}\left(X^{o}\right) .
$$

We now address the questions of existence of an optimal replicating portfolio according to the portfolio selection criterion (2.23), and continuity of the value of the liability cash flow as a function of the portfolio weights of the replicating portfolio. For $t \in\{1, \ldots, T\}$, define

$$
Z_{t}:=\left(X_{t}^{o},-\left(X_{t}^{f}\right)^{\mathrm{T}}\right)^{\mathrm{T}}
$$


and, for $w \in \mathbb{R}^{m+1}, \tilde{X}_{t}^{w}:=w^{\mathrm{T}} Z_{t}$. Notice that a residual liability cash flow corresponds to $\widetilde{X}^{w}$ with $w^{(1)}=1$. The reason for introducing this notation is primarily that it allows us to formulate sufficient conditions for coerciveness that lead to sufficient conditions for the existence of an optimal replicating portfolio; see Theorem 2.28 below.

Theorem 2.27 Let $\left(D_{t}\right)_{t=0}^{T}$ satisfy either of the conditions (i) or (ii) in Theorem 2.17. Suppose that for each $t \in\{0, \ldots, T-1\}, \rho_{t}: L^{1}\left(\mathcal{F}_{t+1}, \mathbb{P}\right) \rightarrow L^{1}\left(\mathcal{F}_{t}, \mathbb{P}\right)$ in (2.14) is a conditional monetary risk measure in the sense of Definition 2.14 such that for every $p \in[1, \infty]$, we have $\rho_{t}(Y) \in L^{p}\left(\mathcal{F}_{t}, \mathbb{P}\right)$ if $Y \in L^{p}\left(\mathcal{F}_{t+1}, \mathbb{P}\right)$, and $\rho_{t}$ is $L^{1}$-Lipschitz-continuous in the sense that

$$
\left|\rho_{t}(-Y)-\rho_{t}(-\tilde{Y})\right| \leq K \mathbb{E}_{t}^{\mathbb{P}}[|Y-\tilde{Y}|], \quad Y, \widetilde{Y} \in L^{1}\left(\mathcal{F}_{t+1}, \mathbb{P}\right),
$$

for some $K \in(0, \infty)$. If $\left(Z_{t}\right)_{t=1}^{T} \in L^{p}\left(\left(\mathcal{F}_{t}\right)_{t=1}^{T}, \mathbb{P}\right)$ for some $p>1$, then

$$
\mathbb{R}^{m+1} \ni w \mapsto \varphi_{0} \circ \cdots \circ \varphi_{T-1}\left(\sum_{t=1}^{T} \tilde{X}_{t}^{w}\right)
$$

and $\mathbb{R}^{m} \ni v \mapsto V_{0}\left(X^{v}\right)$ are Lipschitz-continuous.

Notice that the Lipschitz property of $\rho_{t}$ in Theorem 2.27 holds for $\mathrm{ES}_{t, p}$, but not for $\mathrm{VaR}_{t, p}$. If the latter risk measure is chosen, then conditions need to be imposed on the models for the cash flows in order to establish continuity properties.

For $t=0, \ldots, T-1$, set

$$
\begin{aligned}
\widetilde{V}_{t}^{w} & :=\varphi_{t} \circ \cdots \circ \varphi_{T-1}\left(\sum_{s=t+1}^{T} \widetilde{X}_{s}^{w}\right), \\
\widetilde{R}_{t}^{w} & :=\rho_{t}\left(-\widetilde{X}_{t+1}^{w}-\widetilde{V}_{t+1}^{w}\right), \\
\widetilde{C}_{t}^{w} & :=\mathbb{E}_{t}^{\mathbb{Q}}\left[\left(\widetilde{R}_{t}^{w}-\widetilde{X}_{t+1}^{w}-\widetilde{V}_{t+1}^{w}\right)^{+}\right], \\
\widetilde{\psi}(w) & :=\mathbb{E}_{0}^{\mathbb{Q}}\left[\max _{t \in\{0, \ldots, T-1\}} \widetilde{C}_{t}^{w}\right] .
\end{aligned}
$$

Under mild conditions, it can be shown that $\widetilde{\psi}$ and $\psi$ given by (2.23) are coercive, i.e.,

$$
\lim _{|w| \rightarrow \infty} \tilde{\psi}(w)=\infty, \quad \lim _{|v| \rightarrow \infty} \psi(v)=\infty
$$

Theorem 2.28 Suppose for $t=0, \ldots, T-1$ that $\rho_{t}$ is positively homogeneous in the sense that $\rho_{t}(\lambda Y)=\lambda \rho_{t}(Y)$ for $\lambda \in \mathbb{R}_{+}$. Suppose further that $\inf _{|w|=1} \widetilde{\psi}(w)>0$. Then $\lim _{|w| \rightarrow \infty} \widetilde{\psi}(w)=\infty$ and $\lim _{|v| \rightarrow \infty} \psi(v)=\infty$, where $\psi$ is given by (2.23).

Remark 2.29 Notice that the condition $\inf _{|w|=1} \widetilde{\psi}(w)>0$ means that perfect replication is not possible. It also disqualifies risk-free cash flows as replication instruments. The argument is as follows. If one of the replication instruments has a risk- 
free cash flow $x$ so that $X^{f, k}=x \mathbb{P}$-a.s., then $X^{f, k}=x \mathbb{Q}$-a.s. and $w^{\mathrm{T}} Z=x$ for some $w \in \mathbb{R}^{m+1}$ with $|w|=1$. Then $\widetilde{\psi}(w)=0$.

For $t \in\{0, \ldots, T-1\}$, set

$$
\rho_{t, T-1}^{\circ}:= \begin{cases}\rho_{t}, & t=T-1 \\ \rho_{t} \circ\left(-\rho_{t+1}\right) \circ \cdots \circ\left(-\rho_{T-1}\right), & t<T-1 .\end{cases}
$$

Theorem 2.30 Suppose for $t=0, \ldots, T-1$ that $\rho_{t}$ is positively homogeneous in the sense that $\rho_{t}(\lambda Y)=\lambda \rho_{t}(Y)$ for $\lambda \in \mathbb{R}_{+}$. Suppose further that $\psi$ in (2.23) is continuous and that for all $w \in \mathbb{R}^{m+1} \backslash\{0\}$, there exists $t \in\{0, \ldots, T-1\}$ such that

$$
\mathbb{P}\left[\left(\rho_{t, T-1}^{\circ}-\rho_{t+1, T-1}^{\circ}\right)\left(-w^{\mathrm{T}} \sum_{s=t+1}^{T} Z_{s}\right)>0\right]>0,
$$

where $Z_{t+1}, \ldots, Z_{T}$ are given by $(2.25)$ and $\rho_{T, T-1}^{\circ}\left(-w^{\mathrm{T}} Z_{T}\right):=w^{\mathrm{T}} Z_{T}$. Then there exists a solution $\widehat{v} \in \mathbb{R}^{m}$ to (2.23).

Remark 2.31 The conditions of Theorem 2.30 are sufficient, but not necessary for the existence of a solution to (2.23). For instance, including risk-free cash flows as replication instruments would violate the condition that (2.26) holds for some $t$ and all nonzero $w$ without affecting either the optimal portfolio weights in the original replication instruments or the value of the liability cash flow; see Theorem 2.20.

Condition (2.26) is not very transparent. The following lemma essentially says that (2.26) is satisfied for commonly encountered risk measures whenever the cash flows $Z_{1}, \ldots, Z_{T}$ are linearly independent.

Lemma 2.32 Let $Z_{1}, \ldots, Z_{T}$ be given by (2.25). Consider the following statements:

(i) $w^{\mathrm{T}} \sum_{t=1}^{T} Z_{t} \notin \mathcal{F}_{0}$ for all $w \in \mathbb{R}^{m+1} \backslash\{0\}$,

(ii) $\rho_{t}\left(-w^{\mathrm{T}} \sum_{s=t+1}^{T} Z_{s}\right) \geq \mathbb{E}_{t}^{\mathbb{P}}\left[w^{\mathrm{T}} \sum_{s=t+1}^{T} Z_{s}\right]$ for all $w \in \mathbb{R}^{m+1} \backslash\{0\}$ and $t \in\{0, \ldots, T-1\}$.

Then (i) and (ii) together imply (2.26) for all $w \in \mathbb{R}^{m+1} \backslash\{0\}$ and $t \in\{0, \ldots, T-1\}$.

From Lemma 2.32, it follows that Theorem 2.30 simplifies considerably for the specific choice of conditional risk measure $\mathrm{ES}_{t, p}$.

Corollary 2.33 For $t=0, \ldots, T-1$, suppose $\rho_{t}=\mathrm{ES}_{t, p}$ for some $p \in(0,1)$ and, for all $w \in \mathbb{R}^{m+1} \backslash\{0\}$, that $w^{\mathrm{T}} \sum_{t=1}^{T} Z_{t} \notin \mathcal{F}_{0}$, where $Z_{1}, \ldots, Z_{T}$ are given by (2.25). Then there exists a solution $\widehat{v} \in \mathbb{R}^{m}$ to (2.23).

\section{Gaussian cash flows}

This section serves one purpose: it demonstrates that if the residual cash flow and the processes generating the filtration can be represented by a (possibly multivariate) 
Gaussian process with respect to $\mathbb{P}$, and if the change of measure between $\mathbb{P}$ and $\mathbb{Q}$ is given by a standard Girsanov transformation, then everything can be computed explicitly. It is likely that in many situations, the benefits of having interpretable explicit closed-form expression outweighs the disadvantages of having to impose these rather strong assumptions.

Let $\left(\varepsilon_{t}\right)_{t=1}^{T}$ be a sequence of $n$-dimensional independent random vectors that are standard normally distributed under $\mathbb{P}$. For $t=1, \ldots, T$ and nonrandom $A_{t} \in \mathbb{R}^{n}$, $B_{t, 1}, \ldots, B_{t, t} \in \mathbb{R}^{n \times n}$, let

$$
G_{t}:=A_{t}+\sum_{s=1}^{t} B_{t, s} \varepsilon_{s}
$$

Let $\left(\mathcal{G}_{t}\right)_{t=0}^{T}$, with $\mathcal{G}_{0}=\{\varnothing, \Omega\}$, be the filtration generated by the Gaussian process $\left(G_{t}\right)_{t=1}^{T}$. In what follows, $\mathbb{E}_{t}^{\mathbb{P}}$ and $\mathbb{E}_{t}^{\mathbb{Q}}$ mean conditional expectations with respect to $\mathcal{G}_{t}$. $\left(G_{t}\right)_{t=1}^{T}$, seen as a column vector valued process, is the result of applying an affine transformation $x \mapsto A+B x$ to $\left(\varepsilon_{t}\right)_{t=1}^{T}$, where $B$ is a lower-triangular block matrix with blocks $B_{i, j}$ and determinant $\prod_{t=1}^{T} \operatorname{det}\left(B_{t, t}\right)$. In order to avoid unnecessary technicalities, we assume that $\operatorname{det}\left(B_{t, t}\right) \neq 0$ for all $t$. This implies that the filtration generated by $\left(\varepsilon_{t}\right)_{t=1}^{T}$ equals the filtration generated by $\left(G_{t}\right)_{t=1}^{T}$.

A natural interpretation of the Gaussian model is as follows: $X^{o}=G^{(1)}$ is the discounted liability cash flow, $G^{(2)}, \ldots, G^{(m+1)}$ represent discounted cash flows of replication instruments, and $G^{(m+2)}, \ldots, G^{(n)}$ are application-specific Gaussian processes contributing to the filtration.

For a nonrandom sequence $\left(\lambda_{t}\right)_{t=1}^{T}, \lambda_{t} \in \mathbb{R}^{n}$, let

$$
D_{t}:=\exp \left(\sum_{s=1}^{t}\left(\lambda_{s}^{\mathrm{T}} \varepsilon_{s}-\frac{1}{2} \lambda_{s}^{\mathrm{T}} \lambda_{s}\right)\right), \quad t=1, \ldots, T .
$$

We let the measure $\mathbb{Q}$ be defined in terms of the $(\mathbb{P}, \mathbb{G})$-martingale $\left(D_{t}\right)_{t=1}^{T}$ : for any $\mathcal{G}_{t}$-measurable sufficiently integrable $Z$ and $s<t, \mathbb{E}_{s}^{\mathbb{Q}}[Z]=D_{s}^{-1} \mathbb{E}_{s}^{\mathbb{P}}\left[D_{t} Z\right]$. This choice has several pleasant consequences: for arbitrary vectors $g_{s} \in \mathbb{R}^{n}$ and $u>t$,

$$
\begin{aligned}
& \mathbb{E}_{t}^{\mathbb{Q}}\left[\sum_{s=1}^{u} g_{s}^{\mathrm{T}} G_{s}\right]-\mathbb{E}_{t}^{\mathbb{P}}\left[\sum_{s=1}^{u} g_{s}^{\mathrm{T}} G_{s}\right] \in \mathcal{G}_{0}, \\
& \operatorname{Var}_{t}^{\mathbb{Q}}\left[\sum_{s=1}^{u} g_{s}^{\mathrm{T}} G_{s}\right]=\operatorname{Var}_{t}^{\mathbb{P}}\left[\sum_{s=1}^{u} g_{s}^{\mathrm{T}} G_{s}\right] \in \mathcal{G}_{0},
\end{aligned}
$$

i.e., the conditional expectations with respect to $\mathbb{Q}$ and $\mathbb{P}$ only differ by a constant and the conditional variances with respect to $\mathbb{Q}$ and $\mathbb{P}$ are equal and nonrandom.

Definition 3.1 The triple $\left(\left(G_{t}\right)_{t=1}^{T},\left(D_{t}\right)_{t=1}^{T},\left(\mathcal{G}_{t}\right)_{t=0}^{T}\right)$ as defined above is called a Gaussian model.

The Gaussian model leads to explicit expressions for the quantities $R_{t}, C_{t}$ and $V_{t}$ when combined with conditional monetary risk measures satisfying (2.12). No- 
tice that for $u>t, \sum_{s=1}^{u} g_{s}^{\mathrm{T}} G_{s}-\mathbb{E}_{t}^{\mathbb{P}}\left[\sum_{s=1}^{u} g_{s}^{\mathrm{T}} G_{s}\right]$ is independent of $\mathcal{G}_{t}$, and if $\operatorname{Var}_{t}^{\mathbb{P}}\left[\sum_{s=1}^{u} g_{s}^{\mathrm{T}} G_{s}\right] \neq 0$,

$$
\operatorname{Var}_{t}^{\mathbb{P}}\left[\sum_{s=1}^{u} g_{s}^{\mathrm{T}} G_{s}\right]^{-1 / 2}\left(\sum_{s=1}^{u} g_{s}^{\mathrm{T}} G_{s}-\mathbb{E}_{t}^{\mathbb{P}}\left[\sum_{s=1}^{u} g_{s}^{\mathrm{T}} G_{s}\right]\right)
$$

is standard normally distributed with respect to $\mathbb{P}$. Since a risk measure $\rho_{t}$ satisfying (2.12) has the additional property that $\rho_{t}(\lambda Y)=\lambda \rho_{t}(Y)$ if $\lambda \in \mathbb{R}_{+}$and $Y \in L^{p}\left(\mathcal{F}_{t+1}, \mathbb{P}\right)$ (positive homogeneity), it follows that

$$
\rho_{t}\left(\sum_{s=1}^{u} g_{s}^{\mathrm{T}} G_{s}\right)=-\mathbb{E}_{t}^{\mathbb{P}}\left[\sum_{s=1}^{u} g_{s}^{\mathrm{T}} G_{s}\right]+\operatorname{Var}_{t}^{\mathbb{P}}\left[\sum_{s=1}^{u} g_{s}^{\mathrm{T}} G_{s}\right]^{1 / 2} r_{0},
$$

where

$$
r_{0}:=\int_{0}^{1} \Phi^{-1}(u) d M(u) .
$$

We first derive an explicit expression for the value of a general Gaussian liability cash flow; the generality lies in that $X_{t}$ is allowed to be an arbitrary linear combination $g_{t}^{\mathrm{T}} G_{t}$, where $g_{t} \in \mathbb{R}^{n}$ may be time-dependent. Then we return to the relevant special case when $g_{t}=g$ for all $t$ and $g^{(1)}=1,\left(g^{(k)}\right)_{k=2}^{m+1}=v \in \mathbb{R}^{m}$ and $g^{(k)}=0$ for $k>m+1$.

Theorem 3.2 Let $\left(\left(G_{t}\right)_{t=1}^{T},\left(D_{t}\right)_{t=1}^{T},\left(\mathcal{G}_{t}\right)_{t=0}^{T}\right)$ be a Gaussian model and define the process $X$ by $X_{t}:=g_{t}^{\mathrm{T}} G_{t}$ for $t=1, \ldots, T$. For $t=0, \ldots, T-1$, let $\rho_{t}$ be conditional monetary risk measures satisfying (2.12) for a common probability distribution $M$. Let $r_{0}$ be given by (3.1). Then

$$
V_{t}=\sum_{s=t+1}^{T} \mathbb{E}_{t}^{\mathbb{Q}}\left[X_{s}\right]+K_{t}^{\mathbb{Q}}=\sum_{s=t+1}^{T} \mathbb{E}_{t}^{\mathbb{P}}\left[X_{s}\right]+K_{t}^{\mathbb{P}},
$$

where, with $e_{1}$ standard normally distributed with respect to $\mathbb{P}$,

$$
\begin{aligned}
K_{t}^{\mathbb{Q}} & =\sum_{s=t+1}^{T}\left(\sigma_{s} r_{0}-\sum_{u=s}^{T} g_{u}^{\mathrm{T}} B_{u, s} \lambda_{s}-\mathbb{E}_{0}^{\mathbb{P}}\left[\left(\sigma_{s}\left(r_{0}-e_{1}\right)-\sum_{u=s}^{T} g_{u}^{\mathrm{T}} B_{u, s} \lambda_{s}\right)^{+}\right]\right), \\
K_{t}^{\mathbb{P}} & =\sum_{s=t+1}^{T}\left(\sigma_{s} r_{0}-\mathbb{E}_{0}^{\mathbb{P}}\left[\left(\sigma_{s}\left(r_{0}-e_{1}\right)-\sum_{u=s}^{T} g_{u}^{\mathrm{T}} B_{u, s} \lambda_{s}\right)^{+}\right]\right), \\
\sigma_{s}^{2} & =\operatorname{Var}_{s-1}^{\mathbb{P}}\left[\sum_{u=s}^{T} X_{u}\right]-\operatorname{Var}_{s}^{\mathbb{P}}\left[\sum_{u=s}^{T} X_{u}\right]=\sum_{j=s}^{T} \sum_{k=s}^{T} g_{j}^{\mathrm{T}} B_{j, s} B_{k, s}^{\mathrm{T}} g_{k} .
\end{aligned}
$$

Moreover,

$$
C_{t}:=\rho_{t}\left(-X_{t+1}-V_{t+1}\right)-V_{t}=\mathbb{E}_{0}^{\mathbb{P}}\left[\left(\sigma_{t+1}\left(r_{0}-e_{1}\right)-\sum_{u=t+1}^{T} g_{u}^{\mathrm{T}} B_{u, t+1} \lambda_{t+1}\right)^{+}\right] .
$$


Remark 3.3 Notice that

$$
\begin{aligned}
C_{t} & =\mathbb{E}_{t}^{\mathbb{Q}}\left[\left(\rho_{t}\left(-X_{t+1}-V_{t+1}\right)-X_{t+1}-V_{t+1}\right)^{+}\right] \\
& =\frac{1}{1+\eta_{t}} \mathbb{E}_{t}^{\mathbb{P}}\left[\left(\rho_{t}\left(-X_{t+1}-V_{t+1}\right)-X_{t+1}-V_{t+1}\right)^{+}\right],
\end{aligned}
$$

where, given the setting in Theorem 3.2,

$$
\frac{1}{1+\eta_{t}}=\frac{\mathbb{E}_{0}^{\mathbb{P}}\left[\left(\sigma_{t+1}\left(r_{0}-e_{1}\right)-\sum_{u=t+1}^{T} g_{u}^{\mathrm{T}} B_{u, t+1} \lambda_{t+1}\right)^{+}\right]}{\mathbb{E}_{0}^{\mathbb{P}}\left[\left(\sigma_{t+1}\left(r_{0}-e_{1}\right)\right)^{+}\right]} .
$$

In particular, $\eta_{t} \geq 0$ for every $t$ if $\sum_{u=t+1}^{T} g_{u}^{\mathrm{T}} B_{u, t+1} \lambda_{t+1} \geq 0$ for every $t$. Since

$$
\sum_{u=t+1}^{T} \mathbb{E}_{t}^{\mathbb{Q}}\left[X_{u}\right]-\sum_{u=t+1}^{T} \mathbb{E}_{t}^{\mathbb{P}}\left[X_{u}\right]=\sum_{u=t+1}^{T} \sum_{s=t+1}^{u} g_{u}^{\mathrm{T}} B_{u, s} \lambda_{s}=\sum_{s=t+1}^{T} \sum_{u=s}^{T} g_{u}^{\mathrm{T}} B_{u, s} \lambda_{s},
$$

we see that $\eta_{t} \geq 0$ for every $t$ holds if $\sum_{u=t+1}^{T} \mathbb{E}_{t}^{\mathbb{Q}}\left[X_{u}\right] \geq \sum_{u=t+1}^{T} \mathbb{E}_{t}^{\mathbb{P}}\left[X_{u}\right]$ for every $t$.

The following result presents the value of the liability cash flow when the replicating portfolio is a static portfolio with portfolio weights solving (2.23).

Theorem 3.4 Let $m, n \in \mathbb{N}$ be such that $n \geq 2$ and $1 \leq m \leq n-1$ and consider an $n$-dimensional Gaussian model $\left(\left(G_{t}\right)_{t=1}^{T},\left(D_{t}\right)_{t=1}^{T},\left(\mathcal{G}_{t}\right)_{t=0}^{T}\right)$. Let $X^{o}=G^{(1)}$ denote a discounted liability cash flow and $X^{f, k}:=G^{(k+1)}, k=1, \ldots, m$, discounted cash flows of replication instruments. For $t=0, \ldots, T-1$, let $\rho_{t}$ be conditional monetary risk measures satisfying (2.12) for a common probability distribution $M$. Then there exists a solution to (2.23), and the value of the liability is given by

$$
L_{0}=\sum_{t=1}^{T} \mathbb{E}_{0}^{\mathbb{Q}}\left[X_{t}^{o}\right]+\widehat{K}_{0}^{\mathbb{Q}}
$$

where, with $S_{t}:=\sum_{u=t}^{T} B_{u, t}, e_{1}$ standard normally distributed with respect to $\mathbb{P}$, and $r_{0}$ given by (3.1),

$$
\begin{aligned}
\widehat{K}_{0}^{\mathbb{Q}} & =\sum_{t=1}^{T}\left(\widehat{\sigma}_{t} r_{0}-\widehat{g}^{\mathrm{T}} S_{t} \lambda_{t}-\mathbb{E}_{0}^{\mathbb{P}}\left[\left(\widehat{\sigma}_{t}\left(r_{0}-e_{1}\right)-\widehat{g}^{\mathrm{T}} S_{t} \lambda_{t}\right)^{+}\right]\right), \\
\widehat{\sigma}_{t}^{2} & =\widehat{g}^{\mathrm{T}} S_{t} S_{t}^{\mathrm{T}} \widehat{g}
\end{aligned}
$$

where $\widehat{g}$ is the minimiser in $\left\{g \in \mathbb{R}^{n}: g^{(1)}=1, g^{(k)}=0\right.$ for $\left.k>m+1\right\}$ of

$$
g \mapsto \sum_{t=0}^{T-1} \mathbb{E}_{0}^{\mathbb{P}}\left[\left(\left(g^{\mathrm{T}} S_{t+1} S_{t+1}^{\mathrm{T}} g\right)^{1 / 2}\left(r_{0}-e_{1}\right)-g^{\mathrm{T}} S_{t+1} \lambda_{t+1}\right)^{+}\right]
$$




\section{Conclusions}

We have presented a framework for market-consistent multi-period valuation of liability cash flows in discrete time subject to repeated capital requirements, taking into account capital requirements in terms of conditional monetary risk measures, (partial) replication of the liability cash flow, and limited liability for the owner of the entity managing the run-off of the liability (the reference undertaking). The framework is general in the sense that it allows a wide range of conditional monetary risk measures, arbitrary liability cash flows and arbitrary replication criteria, although we emphasise that the choice of replicating portfolio criterion is important for the valuation framework to have sound economic implications. We advocate choosing the replicating portfolio such that the market-consistent value of the entity managing the liability run-off is minimised. This choice ensures good replication of the liability cash flow when this is possible. To the best of our knowledge, our valuation framework is the first that combines all the above features consistently with the underlying principles of the current regulatory frameworks.

\section{Proofs}

Proof of Theorem 2.8 We first prove that $C_{t}=\mathbb{E}_{t}^{\mathbb{Q}}\left[\left(R_{t}-X_{t+1}-V_{t+1}\right)^{+}\right]$for any $t \in\{0, \ldots, T-1\}$, from which (2.7) follows. We have

$$
\begin{aligned}
& C_{t}=\underset{\tau \in \mathcal{S}_{t+1, T+1}}{\operatorname{ess} \sup } \mathbb{E}_{t}^{\mathbb{Q}}\left[\sum_{s=t+1}^{\tau-1}\left(R_{S-1}-R_{S}-X_{S}\right)\right] \\
& =\operatorname{ess~sup}_{\tau \in \mathcal{S}_{t+1, T+1}} \mathbb{E}_{t}^{\mathbb{Q}}\left[\mathbb{1}_{\{\tau>t+1\}}\left(\sum_{s=t+1}^{\tau-1}\left(R_{s-1}-R_{s}-X_{s}\right)\right)\right] \\
& =\operatorname{ess~sup}_{\tau \in \mathcal{S}_{t+1, T+1}} \mathbb{E}_{t}^{\mathbb{Q}}\left[\mathbb { 1 } _ { \{ \tau > t + 1 \} } \left(\left(R_{t}-R_{t+1}-X_{t+1}\right)\right.\right. \\
& \left.\left.+\mathbb{E}_{t+1}^{\mathbb{Q}}\left[\sum_{s=t+2}^{\tau-1}\left(R_{s-1}-R_{s}-X_{s}\right)\right]\right)\right] \\
& =\underset{A \in \mathcal{F}_{t+1}}{\operatorname{ess} \sup } \mathbb{E}_{t}^{\mathbb{Q}}\left[\mathbb { 1 } _ { A } \left(\left(R_{t}-R_{t+1}-X_{t+1}\right)\right.\right. \\
& \left.\left.+\underset{\tau \in \mathcal{S}_{t+2, T+1}}{\operatorname{ess} \sup } \mathbb{E}_{t+1}^{\mathbb{Q}}\left[\sum_{s=t+2}^{\tau-1}\left(R_{S-1}-R_{S}-X_{S}\right)\right]\right)\right] \\
& =\operatorname{ess} \sup \mathbb{E}_{t}^{\mathbb{Q}}\left[\mathbb{1}_{A}\left(R_{t}-R_{t+1}-X_{t+1}+C_{t+1}\right)\right] \\
& A \in \mathcal{F}_{t+1} \\
& =\underset{A \in \mathcal{F}_{t+1}}{\operatorname{ess} \sup } \mathbb{E}_{t}^{\mathbb{Q}}\left[\mathbb{1}_{A}\left(R_{t}-X_{t+1}-V_{t+1}\right)\right] \\
& =\mathbb{E}_{t}^{\mathbb{Q}}\left[\left(R_{t}-X_{t+1}-V_{t+1}\right)^{+}\right],
\end{aligned}
$$


where we have used the relation $V_{t+1}=R_{t+1}-C_{t+1}$ and that the ess sup in the second to last expression above is attained by choosing $A=\left\{R_{t}-X_{t+1}-V_{t+1} \geq 0\right\}$. Notice that (2.8) now follows immediately from the relation $V_{t}=R_{t}-C_{t}$. Moreover, the sequence of stopping times $\left(\widehat{\tau}_{t}\right)_{t=0}^{T}$ given by $\widehat{\tau}_{T}:=T+1$ and

$$
\widehat{\tau_{t}}:=(t+1) \mathbb{1}_{\left\{R_{t}-X_{t+1}-V_{t+1}<0\right\}}+\widehat{\tau}_{t+1} \mathbb{1}_{\left\{R_{t}-X_{t+1}-V_{t+1} \geq 0\right\}}, \quad t<T,
$$

is optimal. Since $\left(\widehat{\tau}_{t}\right)_{t=0}^{T-1}=\left(\tau_{t}^{*}\right)_{t=0}^{T-1}$, the proof of statements (i) and (ii) is complete.

We now show statement (iii). Let the sequences $\left(C_{t}\right)_{t=0}^{T}$ and $\left(V_{t}\right)_{t=0}^{T}$ be given by (2.7) and (2.8) and the sequences $\left(\widetilde{C}_{t}\right)_{t=0}^{T}$ and $\left(\widetilde{V}_{t}\right)_{t=0}^{T}$ by (2.4) and (2.5). From statement (i), we then know that $\left(\widetilde{C}_{t}\right)_{t=0}^{T}$ and $\left(\widetilde{V}_{t}\right)_{t=0}^{T}$ also satisfy (2.7) and (2.8). Hence $\left(\widetilde{C}_{t}\right)_{t=0}^{T}=\left(C_{t}\right)_{t=0}^{T}$ and $\left(\widetilde{V}_{t}\right)_{t=0}^{T}=\left(V_{t}\right)_{t=0}^{T}$ and thus statement (iii) follows.

The following remark illustrates that Theorem 2.8 can be proved by identifying the cash flows considered here with processes and stopping times that form key ingredients in the framework for valuation of American contingent claims in Föllmer and Schied [12, Chap. 6].

Remark 5.1 Let

$$
\begin{aligned}
H_{t} & :=\sum_{s=1}^{t-1}\left(R_{s-1}-R_{s}-X_{s}\right), \quad t \in\{0, \ldots, T+1\}, \\
U_{T+1} & :=H_{T+1}, \quad U_{t}:=H_{t} \vee \mathbb{E}_{t}^{\mathbb{Q}}\left[U_{t+1}\right], \quad t \in\{0, \ldots, T\} .
\end{aligned}
$$

By [12, Theorem 6.18] and Definitions 2.1 and 2.3,

$$
\begin{aligned}
U_{t} & =\underset{\tau \in \mathcal{S}_{t, T+1}}{\operatorname{ess} \sup } \mathbb{E}_{t}^{\mathbb{Q}}\left[H_{\tau}\right] \\
& =\operatorname{esssup}_{\tau \in \mathcal{S}_{t+1, T+1}} \mathbb{E}_{t}^{\mathbb{Q}}\left[H_{\tau}\right] \vee H_{t} \\
& =\left(H_{t+1}+C_{t}\right) \vee H_{t} \\
& =\left(H_{t}+R_{t-1}-R_{t}-X_{t}+C_{t}\right) \vee H_{t} \\
& =H_{t}+\left(R_{t-1}-X_{t}-V_{t}\right)^{+} .
\end{aligned}
$$

Since $H_{0}=H_{1}=0$, it follows that $U_{0}=C_{0}$. Let

$$
\tau_{\max }^{(t)}:=\min \left\{s \geq t: \mathbb{E}_{s}^{\mathbb{Q}}\left[U_{s+1}\right]<U_{s}\right\} \wedge(T+1), \quad t \in\{0, \ldots, T\} .
$$

By [12, Theorem 6.21], $\tau_{\max }^{(t)}$ is the largest optimal stopping time, i.e., the maximal solution to ess $\sup _{\tau \in \mathcal{S}_{t+1, T+1}} \mathbb{E}_{t}^{\mathbb{Q}}\left[H_{\tau}\right] \vee H_{t}$. We observe, similarly to above, that 
for $s>0$,

$$
\begin{aligned}
\mathbb{E}_{s}^{\mathbb{Q}}\left[U_{s+1}\right]<U_{s} & \Longleftrightarrow \mathbb{E}_{s}^{\mathbb{Q}}\left[U_{s+1}\right]<H_{s} \\
& \Longleftrightarrow H_{s+1}+C_{s}<H_{s} \\
& \Longleftrightarrow R_{s-1}-X_{s}-V_{s}<0 .
\end{aligned}
$$

Similarly, for $s=0, \mathbb{E}^{\mathbb{Q}}\left[U_{1}\right]<U_{0} \Leftrightarrow C_{0}<0$, which is not possible in the current setting. Hence, conditionally on $\left\{\tau_{\max }^{(t)}>t\right\}$, we have $\tau_{\max }^{(t)}=\tau_{t}^{*}$, where $\tau_{t}^{*}$ is defined in Theorem 2.8. With this stopping strategy, conditionally on $\left\{\tau_{\max }^{(t)}>t\right\}$, we obtain

$$
\begin{aligned}
U_{t} & =\left(H_{t}+R_{t-1}-R_{t}-X_{t}+C_{t}\right) \vee H_{t} \\
& =H_{t}+R_{t-1}-R_{t}-X_{t}+C_{t} \\
& =H_{t+1}+C_{t}, \\
U_{t} & =H_{t} \vee \mathbb{E}_{t}^{\mathbb{Q}}\left[U_{t+1}\right] \\
& =\mathbb{E}_{t}^{\mathbb{Q}}\left[U_{t+1}\right] \\
& =\mathbb{E}_{t}^{\mathbb{Q}}\left[H_{t+1}+\left(R_{t}-X_{t+1}-V_{t+1}\right)^{+}\right] \\
& =H_{t+1}+\mathbb{E}_{t}^{\mathbb{Q}}\left[\left(R_{t}-X_{t+1}-V_{t+1}\right)^{+}\right] .
\end{aligned}
$$

This implies (2.7), and therefore also (2.8).

Proof of Theorem 2.17 We prove the more involved statement (ii). Statement (i) is proved with the same arguments. We have

$$
\begin{aligned}
\mathbb{E}^{\mathbb{P}}\left[\mathbb{E}_{t}^{\mathbb{Q}}\left[\left(\rho_{t}(-Y)-Y\right)^{+}\right]^{p}\right] & =\mathbb{E}^{\mathbb{P}}\left[\mathbb{E}_{t}^{\mathbb{P}}\left[\frac{D_{t+1}}{D_{t}}\left(\rho_{t}(-Y)-Y\right)^{+}\right]^{p}\right] \\
& \leq \mathbb{E}^{\mathbb{P}}\left[\mathbb{E}_{t}^{\mathbb{P}}\left[\left(\frac{D_{t+1}}{D_{t}}\right)^{p}\left(\left(\rho_{t}(-Y)-Y\right)^{+}\right)^{p}\right]\right] \\
& =\mathbb{E}^{\mathbb{P}}\left[\left(\frac{D_{t+1}}{D_{t}}\right)^{p}\left(\left(\rho_{t}(-Y)-Y\right)^{+}\right)^{p}\right]
\end{aligned}
$$

where the inequality is due to Jensen's inequality for conditional expectations. Moreover, for every $r>1$, by Hölder's inequality,

$$
\begin{aligned}
& \mathbb{E}^{\mathbb{P}}\left[\left(\frac{D_{t+1}}{D_{t}}\right)^{p}\left(\left(\rho_{t}(-Y)-Y\right)^{+}\right)^{p}\right] \\
& \leq \mathbb{E}^{\mathbb{P}}\left[\left(\frac{D_{t+1}}{D_{t}}\right)^{p r}\right]^{\frac{1}{r}} \mathbb{E}^{\mathbb{P}}\left[\left(\left(\rho_{t}(-Y)-Y\right)^{+}\right)^{p \frac{r}{r-1}}\right]^{\frac{r-1}{r}} .
\end{aligned}
$$


For $r>1$ sufficiently large, it follows from the assumptions that the two expectations exist and are finite. Finally, it follows from Minkowski's inequality that

$$
\begin{aligned}
& \mathbb{E}^{\mathbb{P}}\left[\left(\rho_{t}(-Y)-\mathbb{E}_{t}^{\mathbb{Q}}\left[\left(\rho_{t}(-Y)-Y\right)^{+}\right]\right)^{p}\right]^{\frac{1}{p}} \\
& \leq \mathbb{E}^{\mathbb{P}}\left[\left|\rho_{t}(-Y)\right|^{p}\right]^{\frac{1}{p}}+\mathbb{E}^{\mathbb{P}}\left[\mathbb{E}_{t}^{\mathbb{Q}}\left[\left(\rho_{t}(-Y)-Y\right)^{+}\right]^{p}\right]^{\frac{1}{p}} .
\end{aligned}
$$

The finiteness of the first term follows from the assumptions, and the finiteness of the second term has been proved above. This proves that the mapping is well defined. The remaining part of statement (ii) follows, with minor modifications, from Engsner et al. [10, Proposition 1].

Proof of Theorem 2.19 By Theorem 2.8(iii), it is sufficient to show that for any $t$, we have $X_{t}, \widetilde{R}_{t} \in L^{1}\left(\mathcal{F}_{t}, \mathbb{Q}\right)$. By Theorem 2.17 , there exists $\varepsilon \geq 0$ such that $p-\varepsilon>1$ and $\widetilde{V}_{t+1} \in L^{p-\varepsilon}\left(\mathcal{F}_{t+1}, \mathbb{P}\right)$. Moreover, $X_{t+1} \in L^{p-\varepsilon}\left(\mathcal{F}_{t+1}, \mathbb{P}\right)$. By Theorem 2.16, it now follows that $\widetilde{R}_{t}:=\rho_{t}\left(-X_{t+1}-\widetilde{V}_{t+1}\right) \in L^{p-\varepsilon}\left(\mathcal{F}_{t}, \mathbb{P}\right)$. By Hölder's inequality,

$$
\mathbb{E}_{0}^{\mathbb{Q}}\left[\left|\widetilde{R}_{t}\right|\right]=\mathbb{E}_{0}^{\mathbb{P}}\left[D_{t}\left|\widetilde{R}_{t}\right|\right] \leq \mathbb{E}_{0}^{\mathbb{P}}\left[D_{t}^{r}\right]^{\frac{1}{r}} \mathbb{E}_{0}^{\mathbb{P}}\left[\left|\widetilde{R}_{t}\right|^{\frac{r}{r-1}}\right]^{\frac{r-1}{r}},
$$

where $r$ may be chosen sufficiently large for both factors to exist and be finite. The completely analogous argument for showing $\mathbb{E}_{0}^{\mathbb{Q}}\left[\left|X_{t}\right|\right]<\infty$ is omitted.

Proof of Theorem 2.20 (i) The statement follows immediately from the properties (2.11) and $V_{t}(\tilde{X})=V_{t}(X)-\sum_{s=t+1}^{T} b_{s}$, which is due to (2.18).

(ii) Notice that for all $t \in\{1, \ldots, T\}$, due to (2.15) and (2.17), we have

$$
\begin{aligned}
V_{t}+\sum_{s=1}^{t} X_{s} & =\varphi_{t} \circ \cdots \circ \varphi_{T-1}\left(\sum_{s=t+1}^{T} X_{s}\right)+\sum_{s=1}^{t} X_{s} \\
& =\varphi_{t} \circ \cdots \circ \varphi_{T-1}\left(\sum_{s=1}^{T} X_{s}\right) \\
& =K .
\end{aligned}
$$

Hence for all $t \in\{1, \ldots, T\}, X_{t}+V_{t}=K-\sum_{s=1}^{t-1} X_{s}$ is $\mathcal{F}_{t-1}$-measurable. This in turn, using (2.11), implies that for all $t$,

$$
C_{t}:=\mathbb{E}_{t}^{\mathbb{Q}}\left[\left(\rho_{t}\left(-X_{t+1}-V_{t+1}\right)-X_{t+1}-V_{t+1}\right)^{+}\right]=0 .
$$

(iii) By assumption, for $t \in\{0, \ldots, T-1\}$,

$$
\mathbb{E}_{t}^{\mathbb{Q}}\left[\left(\rho_{t}\left(-X_{t+1}-V_{t+1}\right)-X_{t+1}-V_{t+1}\right)^{+}\right]=0 .
$$

Hence, with $\varphi_{u, v}^{\circ}:=\varphi_{u} \circ \cdots \circ \varphi_{v}$, for $t \in\{0, \ldots, T-1\}$, we have

$$
\mathbb{E}_{t}^{\mathbb{Q}}\left[\left(\rho_{t}\left(-\varphi_{t+1, T-1}^{\circ}\left(\sum_{s=1}^{T} X_{s}\right)\right)-\varphi_{t+1, T-1}^{\circ}\left(\sum_{s=1}^{T} X_{s}\right)\right)^{+}\right]=0,
$$


where $\varphi_{T, T-1}^{\circ}\left(\sum_{s=1}^{T} X_{s}\right)=\sum_{s=1}^{T} X_{s}$. Hence, for $t \in\{0, \ldots, T-1\}$,

$$
\mathbb{P}_{t}\left[\varphi_{t+1, T-1}^{\circ}\left(\sum_{s=1}^{T} X_{s}\right) \geq \rho_{t}\left(-\varphi_{t+1, T-1}^{\circ}\left(\sum_{s=1}^{T} X_{s}\right)\right)\right]=1 .
$$

If $\rho_{t}$ has the property (2.19), then (5.1) implies that $\varphi_{t+1, T-1}^{\circ}\left(\sum_{s=1}^{T} X_{s}\right)$ is $\mathcal{F}_{t}$-measurable. Hence, for $t \in\{0, \ldots, T-1\}$,

$$
\varphi_{t+1, T-1}^{\circ}\left(\sum_{s=1}^{T} X_{S}\right)=\varphi_{t, T-1}^{\circ}\left(\sum_{s=1}^{T} X_{s}\right) .
$$

In particular,

$$
\sum_{s=1}^{T} X_{S}=\varphi_{T, T-1}^{\circ}\left(\sum_{s=1}^{T} X_{S}\right)=\varphi_{0, T-1}^{\circ}\left(\sum_{s=1}^{T} X_{S}\right)=V_{0} .
$$

(iv) and (v) The properties (2.16)-(2.18) together imply that $V_{0} \leq 0$ if $X_{t}^{r} \geq X_{t}^{o}$ for all $t$, and $V_{0} \geq 0$ if $X_{t}^{r} \leq X_{t}^{o}$ for all $t$. The conclusion now follows from the definition of $L_{0}$.

Proof of Corollary 2.21 Consider the representation

$$
\mathrm{ES}_{t, p}(-Y)=\frac{1}{p} \int_{1-p}^{1} F_{t, Y}^{-1}(u) d u .
$$

By Theorem 2.20, it is sufficient to verify the property (2.19) for $\mathrm{ES}_{t, p}$.

If $\mathbb{P}_{t}\left[Y \geq F_{t, Y}^{-1}(1-p)\right]<1$, then

$$
1=\mathbb{P}_{t}\left[Y \geq \operatorname{ES}_{t, p}(-Y)\right] \leq \mathbb{P}_{t}\left[Y \geq F_{t, Y}^{-1}(1-p)\right]<1
$$

which is a contradiction. Hence we have $\mathbb{P}_{t}\left[Y \geq F_{t, Y}^{-1}(1-p)\right]=1$ and consequently $F_{t, Y}^{-1}(q)=F_{t, Y}^{-1}(1-p)$ for all $q \leq 1-p$.

If $F_{t, Y}^{-1}(q)>F_{t, Y}^{-1}(1-p)$ for some $q>1-p$, then $\operatorname{ES}_{t, p}(-Y)>F_{t, Y}^{-1}(1-p)$ and

$$
\begin{aligned}
1= & \mathbb{P}_{t}\left[Y \geq \mathrm{ES}_{t, p}(-Y)\right] \\
= & \mathbb{P}_{t}\left[Y \geq \mathrm{ES}_{t, p}(-Y), Y>F_{t, Y}^{-1}(1-p)\right] \\
& +\mathbb{P}_{t}\left[Y \geq \operatorname{ES}_{t, p}(-Y), Y=F_{t, Y}^{-1}(1-p)\right] \\
= & \mathbb{P}_{t}\left[Y \geq \operatorname{ES}_{t, p}(-Y), Y>F_{t, Y}^{-1}(1-p)\right] \\
\leq & 1-F_{t, Y}\left(F_{t, Y}^{-1}(1-p)\right) \\
\leq & p \\
< & 1
\end{aligned}
$$


which is a contradiction. Hence we have $F_{t, Y}^{-1}(q)=F_{t, Y}^{-1}(1-p)$ for all $q$ which implies that $Y$ is $\mathcal{F}_{t}$-measurable.

Proof of Theorem 2.27 For $w \in \mathbb{R}^{m+1}$ and $t \in\{0, \ldots, T-1\}$, define

$$
V_{t}^{w}:=\varphi_{t} \circ \cdots \circ \varphi_{T-1}\left(\sum_{s=t+1}^{T} w^{\mathrm{T}} Z_{s}\right) .
$$

We prove the statement inductively. Assume that for some $B_{t+2} \in L^{1}\left(\mathcal{F}_{t+2}, \mathbb{P}\right)$ taking nonnegative values, we have

$$
\left|V_{t+1}^{w}-V_{t+1}^{v}\right| \leq\|v-w\|_{1} \mathbb{E}_{t+1}^{\mathbb{P}}\left[B_{t+2}\right],
$$

where $\|\cdot\|_{p}$ denotes the Euclidean $p$-norm in $\mathbb{R}^{m+1}$. We start by showing the induction step, noting that verifying the induction base is trivial since $V_{T}^{w}=0$. Defining $Y_{t+1}^{w}:=w^{\mathrm{T}} Z_{t+1}+V_{t+1}^{w}$ and applying Hölder's inequality gives

$$
\begin{aligned}
\left|Y_{t+1}^{w}-Y_{t+1}^{v}\right| & \leq\left|V_{t+1}^{w}-V_{t+1}^{v}\right|+\left|w^{\mathrm{T}} Z_{t+1}-v^{\mathrm{T}} Z_{t+1}\right| \\
& \leq\|v-w\|_{1} \mathbb{E}_{t+1}^{\mathbb{P}}\left[B_{t+2}\right]+\left|w^{\mathrm{T}} Z_{t+1}-v^{\mathrm{T}} Z_{t+1}\right| \\
& \leq\|v-w\|_{1} \mathbb{E}_{t+1}^{\mathbb{P}}\left[\left\|Z_{t+1}\right\|_{\infty}+B_{t+2}\right] .
\end{aligned}
$$

Now due to the $L^{1}$-Lipschitz-continuity of $\rho_{t}$,

$$
\begin{aligned}
\left|\rho_{t}\left(-Y_{t+1}^{w}\right)-\rho_{t}\left(-Y_{t+1}^{v}\right)\right| & \leq K \mathbb{E}_{t}^{\mathbb{P}}\left[\left|Y_{t+1}^{w}-Y_{t+1}^{v}\right|\right] \\
& \leq K\|v-w\|_{1} \mathbb{E}_{t}^{\mathbb{P}}\left[\left\|Z_{t+1}\right\|_{\infty}+B_{t+2}\right] .
\end{aligned}
$$

With $C_{t}^{w}:=\mathbb{E}_{t}^{\mathbb{Q}}\left[\left(\rho_{t}\left(-Y_{t+1}^{w}\right)-Y_{t+1}^{w}\right)^{+}\right]$, the subadditivity of $x \mapsto x^{+}$yields

$$
\begin{aligned}
C_{t}^{w}-C_{t}^{v} & =\mathbb{E}_{t}^{\mathbb{Q}}\left[\left(\rho_{t}\left(-Y_{t+1}^{w}\right)-Y_{t+1}^{w}\right)^{+}-\left(\rho_{t}\left(-Y_{t+1}^{v}\right)-Y_{t+1}^{v}\right)^{+}\right] \\
& \leq \mathbb{E}_{t}^{\mathbb{Q}}\left[\left(\rho_{t}\left(-Y_{t+1}^{w}\right)-Y_{t+1}^{w}-\rho_{t}\left(-Y_{t+1}^{v}\right)+Y_{t+1}^{v}\right)^{+}\right] \\
& \leq \mathbb{E}_{t}^{\mathbb{Q}}\left[\left|\rho_{t}\left(-Y_{t+1}^{w}\right)-Y_{t+1}^{w}-\rho_{t}\left(-Y_{t+1}^{v}\right)+Y_{t+1}^{v}\right|\right], \\
C_{t}^{w}-C_{t}^{v} & \geq \mathbb{E}_{t}^{\mathbb{Q}}\left[-\left(\rho_{t}\left(-Y_{t+1}^{v}\right)-Y_{t+1}^{v}-\rho_{t}\left(-Y_{t+1}^{w}\right)+Y_{t+1}^{w}\right)^{+}\right] \\
& \geq-\mathbb{E}_{t}^{\mathbb{Q}}\left[\left|\rho_{t}\left(-Y_{t+1}^{w}\right)-Y_{t+1}^{w}-\rho_{t}\left(-Y_{t+1}^{v}\right)+Y_{t+1}^{v}\right|\right],
\end{aligned}
$$

from which it follows that

$$
\begin{aligned}
\left|C_{t}^{w}-C_{t}^{v}\right| & \leq \mathbb{E}_{t}^{\mathbb{Q}}\left[\left|\rho_{t}\left(-Y_{t+1}^{w}\right)-Y_{t+1}^{w}-\rho_{t}\left(-Y_{t+1}^{v}\right)+Y_{t+1}^{v}\right|\right] \\
& \leq\left|\rho_{t}\left(-Y_{t+1}^{w}\right)-\rho_{t}\left(-Y_{t+1}^{v}\right)\right|+\mathbb{E}_{t}^{\mathbb{Q}}\left[\left|Y_{t+1}^{w}-Y_{t+1}^{v}\right|\right] .
\end{aligned}
$$

Moreover, we have

$$
\begin{aligned}
\mathbb{E}_{t}^{\mathbb{Q}}\left[\left|Y_{t+1}^{w}-Y_{t+1}^{v}\right|\right] & \leq \mathbb{E}_{t}^{\mathbb{Q}}\left[\|v-w\|_{1} \mathbb{E}_{t+1}^{\mathbb{P}}\left[\left\|Z_{t+1}\right\|_{\infty}+B_{t+2}\right]\right] \\
& =\|v-w\|_{1} \mathbb{E}_{t}^{\mathbb{P}}\left[\frac{D_{t+1}}{D_{t}} \mathbb{E}_{t+1}^{\mathbb{P}}\left[\left\|Z_{t+1}\right\|_{\infty}+B_{t+2}\right]\right]
\end{aligned}
$$


Hence we get

$$
\begin{aligned}
\left|V_{t}^{w}-V_{t}^{v}\right| \leq & \left|\rho_{t}\left(-Y_{t+1}^{w}\right)-\rho_{t}\left(-Y_{t+1}^{v}\right)\right|+\left|C_{t}^{w}-C_{t}^{v}\right| \\
\leq & 2 K\|v-w\|_{1} \mathbb{E}_{t}^{\mathbb{P}}\left[\left\|Z_{t+1}\right\|_{\infty}+B_{t+2}\right] \\
& +\|v-w\|_{1} \mathbb{E}_{t}^{\mathbb{P}}\left[\frac{D_{t+1}}{D_{t}} \mathbb{E}_{t+1}^{\mathbb{P}}\left[\left\|Z_{t+1}\right\|_{\infty}+B_{t+2}\right]\right] \\
= & \|v-w\|_{1} \mathbb{E}_{t}^{\mathbb{P}}\left[\mathbb{E}_{t+1}^{\mathbb{P}}\left[\left\|Z_{t+1}\right\|_{\infty}+B_{t+2}\right]\left(2 K+\frac{D_{t+1}}{D_{t}}\right)\right] \\
= & \|v-w\|_{1} \mathbb{E}_{t}^{\mathbb{P}}\left[B_{t+1}\right],
\end{aligned}
$$

where

$$
B_{t+1}:=\mathbb{E}_{t+1}^{\mathbb{P}}\left[\left\|Z_{t+1}\right\|_{\infty}+B_{t+2}\right]\left(2 K+\frac{D_{t+1}}{D_{t}}\right), \quad B_{T+1}=0 .
$$

In particular, $\left|V_{0}^{w}-V_{0}^{v}\right| \leq\|v-w\|_{1} \mathbb{E}_{0}^{\mathbb{P}}\left[B_{1}\right]$. Now what remains is to show that $\mathbb{E}_{0}^{\mathbb{P}}\left[B_{1}\right]<\infty$. For the Euclidean norms, the inequality $\|x\|_{p} \leq\|x\|_{1}$ holds for $p \in[1, \infty]$. In particular, for each $t=1, \ldots, T$, we have $0 \leq B_{t} \leq \widetilde{B}_{t}$, where

$$
\widetilde{B}_{t+1}:=\mathbb{E}_{t+1}^{\mathbb{P}}\left[\left\|Z_{t+1}\right\|_{1}+\widetilde{B}_{t+2}\right]\left(2 K+\frac{D_{t+1}}{D_{t}}\right), \quad \widetilde{B}_{T+1}=0 .
$$

Recall that for $t=1, \ldots, T$, all the $Z_{t}^{(k)}$ are in $L^{p_{t}}\left(\mathcal{F}_{t}, \mathbb{P}\right)$ for some $p_{t}>1$. Moreover, notice that if $\widetilde{B}_{t+2} \in L^{q_{t+2}}\left(\mathcal{F}_{t+2}, \mathbb{P}\right)$ for $q_{t+2}>1$, then $\mathbb{E}_{t+1}^{\mathbb{P}}\left[\widetilde{B}_{t+2}\right] \in L^{q_{t+2}}\left(\mathcal{F}_{t+1}, \mathbb{P}\right)$ and, for $r_{t+1}=\min \left(p_{t+1}, q_{t+2}\right)$,

$$
\mathbb{E}_{t+1}^{\mathbb{P}}\left[\left\|Z_{t+1}\right\|_{1}+\widetilde{B}_{t+2}\right] \in L^{r_{t+1}}\left(\mathcal{F}_{t+1}\right) .
$$

Hence, for any $\varepsilon>0$,

$$
\widetilde{B}_{t+1}=\mathbb{E}_{t+1}^{\mathbb{P}}\left[\left\|Z_{t+1}\right\|_{1}+\widetilde{B}_{t+2}\right]\left(2 K+\frac{D_{t+1}}{D_{t}}\right) \in L^{r_{t+1}-\varepsilon}\left(\mathcal{F}_{t+1}\right) .
$$

Since $\widetilde{B}_{T+1}=0$, we may choose $\varepsilon>0$ small enough so that $\widetilde{B}_{t} \in L^{1}\left(\mathcal{F}_{t}, \mathbb{P}\right)$ for $t=1, \ldots, T$. Hence, also $B_{t} \in L^{1}\left(\mathcal{F}_{t}, \mathbb{P}\right)$ for $t=1, \ldots, T$. Finally, notice that $X_{t}^{v}:=X_{t}^{o}-v^{\mathrm{T}} X_{t}^{f}=w^{\mathrm{T}} Z_{t}$ if $w \in \mathbb{R}^{m+1}$ is chosen such that $w^{(1)}=1$ and $\left(w^{(k)}\right)_{k=2}^{m+1}=v$. Therefore, we have also shown that $v \mapsto V_{0}\left(X^{v}\right)$ is Lipschitzcontinuous.

Proof of Theorem 2.28 Positive homogeneity of $\rho_{t}$ implies positive homogeneity of $\varphi_{t}$, which implies $\widetilde{V}_{t}^{w}\left(\lambda \widetilde{X}^{w}\right)=\lambda \widetilde{V}_{t}^{w}\left(\widetilde{X}^{w}\right)$ and further that $\widetilde{\psi}(\lambda w)=\lambda \widetilde{\psi}(w)$. In particular, we can write

$$
\widetilde{\psi}(w)=|w| \widetilde{\psi}(w /|w|) \geq|w| \inf _{|w|=1} \widetilde{\psi}(w)
$$


so that $\lim _{|w| \rightarrow \infty} \widetilde{\psi}(w)=\infty$ follows from $\inf _{|w|=1} \widetilde{\psi}(w)>0$. For the second statement, notice that $X_{t}^{v}:=X_{t}^{o}-v^{\mathrm{T}} X_{t}^{f}=w^{\mathrm{T}} Z_{t}$ if $w \in \mathbb{R}^{m+1}$ is chosen such that we have $w^{(1)}=1$ and $\left(w^{(k)}\right)_{k=2}^{m+1}=v$. Therefore, $\lim _{|w| \rightarrow \infty} \widetilde{\psi}(w)=\infty$ implies $\lim _{|v| \rightarrow \infty} \psi(v)=\infty$.

Proof of Theorem 2.30 Take $w \in \mathbb{R}^{m+1} \backslash\{0\}$. Suppose $\widetilde{C}_{t}^{w}=0 \mathbb{Q}$-a.s. for all $t$. Then $\widetilde{V}_{t}^{w}=\widetilde{R}_{t}^{w} \mathbb{Q}$-a.s. for all $t$ and $\widetilde{R}_{t}^{w}-\widetilde{X}_{t+1}^{w}-\widetilde{R}_{t+1}^{w} \leq 0 \mathbb{Q}$-a.s. for all $t$, which is equivalent to $\widetilde{R}_{t}^{w}-\widetilde{X}_{t+1}^{w}-\widetilde{R}_{t+1}^{w} \leq 0 \mathbb{P}$-a.s. for all $t$ since $\mathbb{P}$ and $\mathbb{Q}$ are equivalent. Notice that

$$
\begin{aligned}
\widetilde{R}_{t}^{w} & =\rho_{t}\left(-\widetilde{X}_{t+1}^{w}-\widetilde{R}_{t+1}^{w}\right)=\rho_{t}\left(-\widetilde{X}_{t+1}^{w}-\rho_{t+1}\left(-\widetilde{X}_{t+2}^{w}-\widetilde{R}_{t+2}^{w}\right)\right) \\
& =\rho_{t} \circ\left(-\rho_{t+1}\right) \circ \cdots \circ\left(-\rho_{T-1}\right)\left(-\sum_{s=t+1}^{T} \widetilde{X}_{s}^{w}\right) .
\end{aligned}
$$

The inequality $\widetilde{R}_{t}^{w}-\widetilde{X}_{t+1}^{w}-\widetilde{R}_{t+1}^{w} \leq 0 \mathbb{P}$-a.s. can thus be expressed as

$$
\left(\rho_{t, T-1}^{\circ}-\rho_{t+1, T-1}^{\circ}\right)\left(-w^{\mathrm{T}}\left(Z_{t+1}+\cdots+Z_{T}\right)\right) \leq 0 \quad \mathbb{P} \text {-a.s. }
$$

However, this contradicts the assumption in the statement of the theorem. Therefore we conclude that $\widetilde{C}_{t}^{w}>0 \mathbb{Q}$-a.s. for some $t$, which implies that $\widetilde{\psi}(w)>0$. Therefore, by Theorem 2.28, $\psi$ is coercive; so if a minimum exists, it exists in some compact set in $\mathbb{R}^{m}$. However, a continuous function on a compact set attains its infimum.

Proof of Lemma 2.32 We prove the statement by proving the reversed implication: given (ii), if there exists some $w$ such that (2.26) does not hold for any $t$, then (i) does not hold.

Assume that (ii) holds and there exists some $w$ such that (2.26) does not hold for any $t$. We prove by induction that $w^{\mathrm{T}}\left(Z_{t+1}+\cdots+Z_{T}\right) \in \mathcal{F}_{t}$ for $t=0, \ldots, T-1$.

Induction base: Since (2.26) does not hold, $\rho_{T-1}\left(-w^{\mathrm{T}} Z_{T}\right) \leq w^{\mathrm{T}} Z_{T}$. However, due to (ii), $\rho_{T-1}\left(-w^{\mathrm{T}} Z_{T}\right) \geq \mathbb{E}_{T-1}^{\mathbb{P}}\left[w^{\mathrm{T}} Z_{T}\right]$. Therefore we get

$$
w^{\mathrm{T}} Z_{T}=\rho_{T-1}\left(-w^{\mathrm{T}} Z_{T}\right) \in \mathcal{F}_{T-1} .
$$

Induction step: Assume that $w^{\mathrm{T}}\left(Z_{t+2}+\cdots+Z_{T}\right) \in \mathcal{F}_{t+1}$. Since (2.26) does not hold, $\left(\rho_{t, T-1}^{\circ}-\rho_{t+1, T-1}^{\circ}\right)\left(-w^{\mathrm{T}}\left(Z_{t+1}+\cdots+Z_{T}\right)\right) \leq 0$. Using the translation invariance property (2.11) of the $\rho_{t}$, the inequality simplifies to

$$
\rho_{t}\left(-w^{\mathrm{T}}\left(Z_{t+1}+\cdots+Z_{T}\right)\right) \leq w^{\mathrm{T}}\left(Z_{t+1}+\cdots+Z_{T}\right) .
$$

Then analogously to the proof for the induction base, (ii) implies that

$$
w^{\mathrm{T}}\left(Z_{t+1}+\cdots+Z_{T}\right)=\rho_{t}\left(-w^{\mathrm{T}}\left(Z_{t+1}+\cdots+Z_{T}\right)\right) \in \mathcal{F}_{t},
$$

which completes the proof of the induction step.

Using the proved result for $t=0$ yields $w^{\mathrm{T}}\left(Z_{1}+\cdots+Z_{T}\right) \in \mathcal{F}_{0}$, i.e., (i) does not hold. 
Proof of Corollary 2.33 Note that expected shortfall is Lipschitz-continuous and for any $p$ satisfies the property $\operatorname{ES}_{t, p}(-X) \geq \mathbb{E}_{t}[X]$. The conclusion then immediately follows from combining Theorems 2.27, 2.28, 2.30 and Lemma 2.32.

Lemma 5.2 For $u<v, \mathbb{E}_{u}^{\mathbb{Q}}\left[G_{v}\right]=\mathbb{E}_{u}^{\mathbb{P}}\left[G_{v}\right]+\sum_{s=u+1}^{v} B_{v, s} \lambda_{s}$.

Proof

$$
\begin{aligned}
\mathbb{E}_{u}^{\mathbb{Q}}\left[G_{v}\right] & =A_{v}+\sum_{s=1}^{u} B_{v, s} \varepsilon_{s}+\sum_{s=u+1}^{v} B_{v, s} \mathbb{E}_{u}^{\mathbb{P}}\left[\frac{D_{v}}{D_{u}} \varepsilon_{s}\right] \\
& =A_{v}+\sum_{s=1}^{u} B_{v, s} \varepsilon_{s}+\sum_{s=u+1}^{v} B_{v, s} \mathbb{E}_{0}^{\mathbb{P}}\left[\exp \left(\lambda_{s}^{\mathrm{T}} \varepsilon_{1}-\frac{1}{2} \lambda_{s}^{\mathrm{T}} \lambda_{s}\right) \varepsilon_{1}\right] \\
& =A_{v}+\sum_{s=1}^{u} B_{v, s} \varepsilon_{s}+\sum_{s=u+1}^{v} B_{v, s} \lambda_{s} \\
& =\mathbb{E}_{u}^{\mathbb{P}}\left[G_{v}\right]+\sum_{s=u+1}^{v} B_{v, s} \lambda_{s} .
\end{aligned}
$$

Lemma 5.3 If $X_{s}:=g_{s}^{\mathrm{T}} G_{s}$ for all $s$, then

$$
\mathbb{E}_{t}^{\mathbb{P}}\left[\mathbb{E}_{t+1}^{\mathbb{Q}}\left[\sum_{s=t+1}^{T} X_{s}\right]\right]=\mathbb{E}_{t}^{\mathbb{Q}}\left[\sum_{s=t+1}^{T} X_{s}\right]-\sum_{s=t+1}^{T} g_{s}^{\mathrm{T}} B_{s, t+1} \lambda_{t+1}
$$

Proof For $s \geq t+1$, with an empty sum defined as 0, it follows from Lemma 5.2 that

$$
\begin{aligned}
& \mathbb{E}_{t+1}^{\mathbb{Q}}\left[X_{s}\right]=\mathbb{E}_{t+1}^{\mathbb{P}}\left[X_{s}\right]+g_{s}^{\mathrm{T}} \sum_{u=t+2}^{s} B_{s, u} \lambda_{u}, \\
& \mathbb{E}_{t}^{\mathbb{P}}\left[\mathbb{E}_{t+1}^{\mathbb{Q}}\left[\sum_{s=t+1}^{T} X_{s}\right]\right]=\sum_{s=t+1}^{T}\left(\mathbb{E}_{t}^{\mathbb{P}}\left[\mathbb{E}_{t+1}^{\mathbb{P}}\left[X_{s}\right]\right]+g_{s}^{\mathrm{T}} \sum_{u=t+2}^{s} B_{s, u} \lambda_{u}\right) \\
& =\mathbb{E}_{t}^{\mathbb{P}}\left[\sum_{s=t+1}^{T} X_{s}\right]+\sum_{s=t+1}^{T} g_{s}^{\mathrm{T}} \sum_{u=t+2}^{s} B_{s, u} \lambda_{u}, \\
& \mathbb{E}_{t}^{\mathbb{P}}\left[\sum_{s=t+1}^{T} X_{s}\right]=\mathbb{E}_{t}^{\mathbb{Q}}\left[\sum_{s=t+1}^{T} X_{s}\right]-\sum_{s=t+1}^{T} g_{s}^{\mathrm{T}} \sum_{u=t+1}^{s} B_{s, u} \lambda_{u} .
\end{aligned}
$$

Proof of Theorem 3.2 We prove inductively that

$$
V_{t}=\mathbb{E}_{t}^{\mathbb{Q}}\left[\sum_{s=t+1}^{T} X_{s}\right]+K_{t}^{\mathbb{Q}},
$$


and derive the recursive form of the constant term $K_{t}^{\mathbb{Q}}$ via induction. The induction base is trivial: $V_{T}=0$. Now assume that (5.2) holds for $t+1$. Notice that

$$
\begin{aligned}
V_{t}= & \varphi_{t}\left(X_{t+1}+\mathbb{E}_{t+1}^{\mathbb{Q}}\left[\sum_{s=t+2}^{T} X_{s}\right]+K_{t+1}^{\mathbb{Q}}\right) \\
= & \varphi_{t}\left(\mathbb{E}_{t+1}^{\mathbb{Q}}\left[\sum_{s=t+1}^{T} X_{s}\right]+K_{t+1}^{\mathbb{Q}}\right) \\
= & K_{t+1}^{\mathbb{Q}}+\rho_{t}\left(-\mathbb{E}_{t+1}^{\mathbb{Q}}\left[\sum_{s=t+1}^{T} X_{s}\right]\right) \\
& -\mathbb{E}_{t}^{\mathbb{Q}}\left[\left(\rho_{t}\left(-\mathbb{E}_{t+1}^{\mathbb{Q}}\left[\sum_{s=t+1}^{T} X_{s}\right]\right)-\mathbb{E}_{t+1}^{\mathbb{Q}}\left[\sum_{s=t+1}^{T} X_{s}\right]\right)^{+}\right] .
\end{aligned}
$$

We first evaluate the risk measure part; this is

$$
\begin{aligned}
& \rho_{t}\left(-\mathbb{E}_{t+1}^{\mathbb{Q}}\left[\sum_{s=t+1}^{T} X_{s}\right]\right) \\
& =\mathbb{E}_{t}^{\mathbb{P}}\left[\mathbb{E}_{t+1}^{\mathbb{Q}}\left[\sum_{s=t+1}^{T} X_{s}\right]\right]+\operatorname{Var}_{t}^{\mathbb{P}}\left[\mathbb{E}_{t+1}^{\mathbb{Q}}\left[\sum_{s=t+1}^{T} X_{s}\right]\right]^{1 / 2} r_{0} \\
& =\mathbb{E}_{t}^{\mathbb{Q}}\left[\sum_{s=t+1}^{T} X_{s}\right]-\sum_{s=t+1}^{T} g_{s}^{\mathrm{T}} B_{s, t+1} \lambda_{t+1}+\operatorname{Var}_{t}^{\mathbb{P}}\left[\mathbb{E}_{t+1}^{\mathbb{Q}}\left[\sum_{s=t+1}^{T} X_{s}\right]\right]^{1 / 2} r_{0},
\end{aligned}
$$

where the final step uses Lemma 5.3. Moreover,

$$
\begin{aligned}
\operatorname{Var}_{t}^{\mathbb{P}}\left[\mathbb{E}_{t+1}^{\mathbb{Q}}\left[\sum_{s=t+1}^{T} X_{s}\right]\right] & =\operatorname{Var}_{t}^{\mathbb{Q}}\left[\mathbb{E}_{t+1}^{\mathbb{Q}}\left[\sum_{s=t+1}^{T} X_{s}\right]\right] \\
& =\operatorname{Var}_{t}^{\mathbb{Q}}\left[\sum_{s=1}^{T} X_{s}\right]-\operatorname{Var}_{t+1}^{\mathbb{Q}}\left[\sum_{s=1}^{T} X_{s}\right]=: \sigma_{t+1}^{2} .
\end{aligned}
$$

For the remaining term, if $\sigma_{t+1} \neq 0$, there exists a random variable $e_{t+1}^{*}$ independent of $\mathcal{G}_{t}$ and standard normally distributed with respect to $\mathbb{Q}$ such that

$$
\begin{aligned}
& \mathbb{E}_{t}^{\mathbb{Q}}\left[\left(\rho_{t}\left(-\mathbb{E}_{t+1}^{\mathbb{Q}}\left[\sum_{s=t+1}^{T} X_{s}\right]-K_{t+1}^{\mathbb{Q}}\right)-\mathbb{E}_{t+1}^{\mathbb{Q}}\left[\sum_{s=t+1}^{T} X_{s}\right]-K_{t+1}^{\mathbb{Q}}\right)^{+}\right] \\
& =\mathbb{E}_{t}^{\mathbb{Q}}\left[\left(\sigma_{t+1} r_{0}-\sum_{s=t+1}^{T} g_{s}^{\mathrm{T}} B_{s, t+1} \lambda_{t+1}-\sigma_{t+1} e_{t+1}^{*}\right)^{+}\right] \\
& =\mathbb{E}_{0}^{\mathbb{P}}\left[\left(\sigma_{t+1} r_{0}-\sum_{s=t+1}^{T} g_{s}^{\mathrm{T}} B_{s, t+1} \lambda_{t+1}-\sigma_{t+1} e_{1}\right)^{+}\right] .
\end{aligned}
$$


Putting the pieces together now yields

$$
\begin{aligned}
V_{t}= & \mathbb{E}_{t}^{\mathbb{Q}}\left[\sum_{s=t+1}^{T} X_{s}\right]+K_{t+1}^{\mathbb{Q}}+\sigma_{t+1} r_{0}-\sum_{s=t+1}^{T} g_{s}^{\mathrm{T}} B_{s, t+1} \lambda_{t+1} \\
& -\mathbb{E}_{0}^{\mathbb{P}}\left[\left(\sigma_{t+1}\left(r_{0}-e_{1}\right)-\sum_{s=t+1}^{T} g_{s}^{\mathrm{T}} B_{s, t+1} \lambda_{t+1}\right)^{+}\right],
\end{aligned}
$$

which proves the induction step and implies that

$$
K_{t}^{\mathbb{Q}}=\sum_{s=t+1}^{T}\left(\sigma_{s} r_{0}-\sum_{u=s}^{T} g_{u}^{\mathrm{T}} B_{u, s} \lambda_{s}-\mathbb{E}_{0}^{\mathbb{P}}\left[\left(\sigma_{s}\left(r_{0}-e_{1}\right)-\sum_{u=s}^{T} g_{u}^{\mathrm{T}} B_{u, s} \lambda_{s}\right)^{+}\right]\right) .
$$

Finally,

$$
\begin{aligned}
V_{t} & =\mathbb{E}_{t}^{\mathbb{Q}}\left[\sum_{s=t+1}^{T} X_{s}\right]+K_{t}^{\mathbb{Q}} \\
& =\mathbb{E}_{t}^{\mathbb{P}}\left[\sum_{s=t+1}^{T} X_{s}\right]+\sum_{s=t+1}^{T} \sum_{u=t+1}^{s} g_{s}^{\mathrm{T}} B_{s, u} \lambda_{u}+K_{t}^{\mathbb{Q}} \\
& =\mathbb{E}_{t}^{\mathbb{P}}\left[\sum_{s=t+1}^{T} X_{s}\right]+K_{t}^{\mathbb{P}},
\end{aligned}
$$

where $K_{t}^{\mathbb{P}}=\sum_{s=t+1}^{T}\left(\sigma_{s} r_{0}-\mathbb{E}_{0}^{\mathbb{P}}\left[\left(\sigma_{s}\left(r_{0}-e_{1}\right)-\sum_{u=s}^{T} g_{u}^{\mathrm{T}} B_{u, s} \lambda_{s}\right)^{+}\right]\right)$. We now derive an expression for $\sigma_{t+1}$. Recall that $X_{s}:=g_{s}^{\mathrm{T}} G_{s}$. Then

$$
\begin{aligned}
\operatorname{Var}_{t}^{\mathbb{P}}\left[\sum_{s=t+1}^{T} g_{s}^{\mathrm{T}} G_{s}\right] & =\operatorname{Var}_{t}^{\mathbb{P}}\left[\sum_{s=t+1}^{T} \sum_{u=t+1}^{s} g_{s}^{\mathrm{T}} B_{s, u} \varepsilon_{u}\right] \\
& =\operatorname{Var}_{t}^{\mathbb{P}}\left[\sum_{u=t+1}^{T} \sum_{s=u}^{T} g_{s}^{\mathrm{T}} B_{s, u} \varepsilon_{u}\right] \\
& =\sum_{u=t+1}^{T} \operatorname{Var}_{t}^{\mathbb{P}}\left[\sum_{s=u}^{T} g_{s}^{\mathrm{T}} B_{s, u} \varepsilon_{u}\right] \\
& =\sum_{u=t+1}^{T}\left(\sum_{s=u}^{T} g_{s}^{\mathrm{T}} B_{s, u}\right)\left(\sum_{s=u}^{T} g_{s}^{\mathrm{T}} B_{s, u}\right)^{\mathrm{T}} \\
& =\sum_{u=t+1}^{T} \sum_{j=u}^{T} \sum_{k=u}^{T} g_{j}^{\mathrm{T}} B_{j, u} B_{k, u}^{\mathrm{T}} g_{k}
\end{aligned}
$$


and

$$
\begin{aligned}
\sigma_{t+1}^{2} & :=\operatorname{Var}_{t}^{\mathbb{P}}\left[\sum_{s=t+1}^{T} g_{s}^{\mathrm{T}} G_{s}\right]-\operatorname{Var}_{t+1}^{\mathbb{P}}\left[\sum_{s=t+1}^{T} g_{s}^{\mathrm{T}} G_{s}\right] \\
& =\sum_{j=t+1}^{T} \sum_{k=t+1}^{T} g_{j}^{\mathrm{T}} B_{j, t+1} B_{k, t+1}^{\mathrm{T}} g_{k} .
\end{aligned}
$$

We now derive the expression for $C_{t}$. Using the same arguments as earlier in the proof,

$$
\begin{aligned}
C_{t} & =\rho_{t}\left(-X_{t+1}-V_{t+1}\right)-V_{t} \\
& =\rho_{t}\left(-\mathbb{E}_{t+1}^{\mathbb{Q}}\left[\sum_{s=t+1}^{T} X_{s}\right]-K_{t+1}^{\mathbb{Q}}\right)-\mathbb{E}_{t}^{\mathbb{Q}}\left[\sum_{s=t+1}^{T} X_{s}\right]-K_{t}^{\mathbb{Q}} \\
& =\sigma_{t+1} r_{0}-\sum_{s=t+1}^{T} g_{s}^{\mathrm{T}} B_{s, t+1} \lambda_{t+1}-K_{t}^{\mathbb{Q}}+K_{t+1}^{\mathbb{Q}} \\
& =\mathbb{E}_{0}^{\mathbb{P}}\left[\left(\sigma_{t+1}\left(r_{0}-e_{1}\right)-\sum_{s=t+1}^{T} g_{s}^{\mathrm{T}} B_{s, t+1} \lambda_{t+1}\right)^{+}\right] .
\end{aligned}
$$

Proof of Theorem 3.4 We prove that there exists a solution to (2.23). The remaining part then follows from Theorem 3.2.

From Theorem 3.2, we immediately see that $\psi$ is continuous. Once we show that for all $w \in \mathbb{R}^{m+1} \backslash\{0\}$, there exists $t \in\{0, \ldots, T-1\}$ such that (2.26) holds, existence of a solution to (2.23) follows. We prove this statement by first proving that there is no $w \in \mathbb{R}^{m+1} \backslash\{0\}$ such that $\sum_{t=1}^{T} w^{\mathrm{T}} Z_{t} \in \mathcal{G}_{0}$, where $Z_{t}:=\left(X_{t}^{o},-\left(X_{t}^{f}\right)^{\mathrm{T}}\right)^{\mathrm{T}}$. Notice that for $g \in \mathbb{R}^{n}$, we have

$$
g^{\mathrm{T}} \sum_{t=1}^{T} G_{t}=g^{\mathrm{T}} \sum_{t=1}^{T} A_{t}+g^{\mathrm{T}} \sum_{s=1}^{T-1} \sum_{t=s}^{T} B_{t, s} \varepsilon_{s}+g^{\mathrm{T}} B_{T, T} \varepsilon_{T} .
$$

The $\varepsilon_{s}$ are independent and $g^{\mathrm{T}} B_{T, T} \neq 0$ for all $g \neq 0$. Hence there is no $g \in \mathbb{R}^{n} \backslash\{0\}$ such that $g^{\mathrm{T}} \sum_{t=1}^{T} G_{t} \in \mathcal{G}_{0}$, which in turn implies that there is no $w \in \mathbb{R}^{m+1} \backslash\{0\}$ such that $\sum_{t=1}^{T} w^{\mathrm{T}} Z_{t} \in \mathcal{G}_{0}$. We now prove that the latter statement implies that for all $w \in \mathbb{R}^{m+1} \backslash\{0\}$, there exists $t \in\{0, \ldots, T-1\}$ such that (2.26) holds.

Notice that

$$
\begin{aligned}
& \left(\rho_{t, T-1}^{\circ}-\rho_{t+1, T-1}^{\circ}\right)\left(-w^{\mathrm{T}}\left(Z_{t+1}+\cdots+Z_{T}\right)\right) \\
& =\left(\rho_{t, T-1}^{\circ}-\rho_{t+1, T-1}^{\circ}\right)\left(-w^{\mathrm{T}}\left(Z_{1}+\cdots+Z_{T}\right)\right) \\
& =\mathbb{E}_{t}^{\mathbb{P}}\left[w^{\mathrm{T}}\left(Z_{1}+\cdots+Z_{T}\right)\right]-\mathbb{E}_{t+1}^{\mathbb{P}}\left[w^{\mathrm{T}}\left(Z_{1}+\cdots+Z_{T}\right)\right]+c
\end{aligned}
$$


for some constant $c$, where the last equality follows from calculations completely analogous to the proof of Theorem 3.2. Now assume that for some $w \in \mathbb{R}^{m+1} \backslash\{0\}$, (2.26) does not hold. In the current Gaussian setting, the support of a Gaussian distribution is either infinite or a singleton. So $\left(\rho_{t, T-1}^{\circ}-\rho_{t+1, T-1}^{\circ}\right)\left(-w^{\mathrm{T}}\left(Z_{1}+\cdots+Z_{T}\right)\right)=0 \mathbb{P}$-a.s. for all $t$ or, equivalently,

$$
\mathbb{E}_{t}^{\mathbb{P}}\left[w^{\mathrm{T}}\left(Z_{1}+\cdots+Z_{T}\right)\right]-\mathbb{E}_{t+1}^{\mathbb{P}}\left[w^{\mathrm{T}}\left(Z_{1}+\cdots+Z_{T}\right)\right] \in \mathcal{G}_{0} \quad \text { for all } t .
$$

For $t=0,(5.3)$ implies that $\mathbb{E}_{1}^{\mathbb{P}}\left[w^{\mathrm{T}}\left(Z_{1}+\cdots+Z_{T}\right)\right] \in \mathcal{G}_{0}$ which together with (5.3) for $t=1$ implies that $\mathbb{E}_{2}^{\mathbb{P}}\left[w^{\mathrm{T}}\left(Z_{1}+\cdots+Z_{T}\right)\right] \in \mathcal{G}_{0}$. By repeating this argument, we obtain that $w^{\mathrm{T}}\left(Z_{1}+\cdots+Z_{T}\right)=\mathbb{E}_{T}^{\mathbb{P}}\left[w^{\mathrm{T}}\left(Z_{1}+\cdots+Z_{T}\right)\right] \in \mathcal{G}_{0}$, which contradicts the assumption $w^{\mathrm{T}}\left(Z_{1}+\cdots+Z_{T}\right) \notin \mathcal{G}_{0}$. Hence we conclude that there exists a solution to $(2.23)$.

Acknowledgements Open access funding provided by Stockholm University. The authors thank the editors and anonymous reviewers for comments, suggestions and criticism that led to a substantial improvement of the paper.

Publisher's Note Springer Nature remains neutral with regard to jurisdictional claims in published maps and institutional affiliations.

Open Access This article is distributed under the terms of the Creative Commons Attribution 4.0 International License (http://creativecommons.org/licenses/by/4.0/), which permits unrestricted use, distribution, and reproduction in any medium, provided you give appropriate credit to the original author(s) and the source, provide a link to the Creative Commons license, and indicate if changes were made.

\section{References}

1. Artzner, P., Delbaen, F., Eber, J.-M., Heath, D., Ku, H.: Coherent multiperiod risk adjusted values and Bellman's principle. Ann. Oper. Res. 152, 5-22 (2007)

2. Barigou, K., Dhaene, J.: Fair valuation of insurance liabilities via mean-variance hedging in a multi period setting. Scand. Actuar. J. 2019, 163-187 (2019)

3. Bion-Nadal, J.: Dynamic risk measures: time consistency and risk measures from BMO martingales. Finance Stoch. 12, 219-244 (2008)

4. Cambou, M., Filipović, D.: Replicating portfolio approach to capital calculation. Finance Stoch. 22, 181-203 (2018)

5. Černý, A., Kallsen, J.: Hedging by sequential regression revisited. Math. Finance 19, 591-617 (2009)

6. Cheridito, P., Kupper, M.: Recursiveness of indifference prices and translation-invariant preferences. Math. Financ. Econ. 2, 173-188 (2009)

7. Cheridito, P., Kupper, M.: Composition of time-consistent dynamic monetary risk measures in discrete time. Int. J. Theor. Appl. Finance 14, 137-162 (2011)

8. Cheridito, P., Kupper, M., Delbaen, F.: Dynamic monetary risk measures for bounded discrete-time processes. Electron. J. Probab. 11, 57-106 (2006)

9. Detlefsen, K., Scandolo, G.: Conditional and dynamic convex risk measures. Finance Stoch. 9, 539$561(2005)$

10. Engsner, H., Lindholm, M., Lindskog, F.: Insurance valuation: a computable multi-period cost-ofcapital approach. Insur. Math. Econ. 72, 250-264 (2017)

11. European Commission: Commission Delegated Regulation (EU) 2015/35 of 10 October 2014. Official Journal of the European Union (2015). Available online at https://eur-lex.europa.eu/legal-content/ EN/TXT/?uri=OJ:L:2015:012:TOC

12. Föllmer, H., Schied, A.: Stochastic Finance: An Introduction in Discrete Time, 4th edn. Walter de Gruyter, Berlin (2016) 
13. Grosen, A., Jørgensen, P.L.: Life insurance liabilities at market value: an analysis of insolvency risk, bonus policy, and regulatory intervention rules in a barrier option framework. J. Risk Insur. 69, 63-91 (2002)

14. Hancock, J., Huber, P., Koch, P.: Value creation in the insurance industry. Risk Manag. Insur. Rev. 4(2), 1-9 (2001)

15. Happ, S., Merz, M., Wüthrich, M.V.: Best-estimate claims reserves in incomplete markets. Eur. Actuar. J. 5, 55-77 (2015)

16. Heath, D., Platen, E., Schweizer, M.: A comparison of two quadratic approaches to hedging in incomplete markets. Math. Finance 11, 385-413 (2001)

17. Hieber, P., Natolski, J., Werner, R.: Fair valuation of cliquet-style return guarantees in (homogeneous and) heterogeneous life insurance portfolios. Scand. Actuar. J. 2019, 478-507 (2019)

18. Malamud, S., Trubowitz, E., Wüthrich, M.V.: Market consistent pricing of insurance products. ASTIN Bull. 38, 483-526 (2008)

19. Möhr, C.: Market-consistent valuation of insurance liabilities by cost of capital. ASTIN Bull. 41, 315-341 (2011)

20. Natolski, J., Werner, R.: Mathematical analysis of different approaches to replicating portfolios. Eur. Actuar. J. 4, 411-435 (2014)

21. Natolski, J., Werner, R.: Mathematical analysis of replication by cash flow matching. Risks 5, 13 (2017)

22. Natolski, J., Werner, R.: Mathematical foundation of the replicating portfolio approach. Scand. Actuar. J. 2018, 481-504 (2018)

23. Pelsser, A., Stadje, M.: Time-consistent and market-consistent evaluations. Math. Finance 24, 25-65 (2014)

24. Schweizer, M.: From actuarial to financial valuation principles. Insur. Math. Econ. 28, 31-47 (2001)

25. Schweizer, M.: Local risk-minimization for multidimensional assets and payment streams. Banach Cent. Publ. 83, 213-229 (2008)

26. Schweizer, M.: Mean-variance hedging. In: Cont, R. (ed.) Encyclopedia of Quantitative Finance, pp. 1177-1181. Wiley, New York (2010)

27. Tsanakas, A., Wüthrich, M.V., Černý, A.: Market value margin via mean-variance hedging. ASTIN Bull. 43, 301-322 (2013) 